\title{
VERMA MODULES AND DIFFERENTIAL CONFORMAL INVARIANTS
}

\author{
R. J. BASTON
}

\begin{abstract}
A distinguished class of differential invariants of conformal structures is constructed using homomorphisms of Verma modules and Cartan's conformal connection. This class reduces to a large subset of the class of differential operators invariant by conformal translations on flat conformal manifolds and operators in it may be expressed in terms of the Levi-Civita connection and Ricci curvature of a metric on the conformal class. Composition of these differential operators yields further differential invariants which depend on the conformal curvature. In all even dimensions, a tracefree symmetric rank two tensor is obtained which is an analogue of the conformally invariant Bach tensor in four dimensions, as a consequence of the structure theory of Verma modules.
\end{abstract}

\section{Introduction}

The invariant linear differential operators on a Riemannian manifold which depend polynomially on the metric $g$, finitely many of its derivatives, $(\operatorname{det} g)^{-1}$, and finitely many derivatives of a spinor or tensor field are well understood. They are obtained by repeated applications of the LeviCivita connection, symmetrization, antisymmetrization, and contraction, a result which follows from the classical invariant theory of the orthogonal group. This characterization is a key ingredient in the proof of the Index Theorem by heat kernel methods [1].

The situation is less clear for such operators on a conformal manifold. Several partial results are known, with many examples [10], [20], [21], [37], and when the conformal structure is flat, a complete theory of such operators exists [17], [26] using result from representation theory on homomorphisms of Verma modules. The purpose of this paper is to give an extension of this theory in the curved case using Cartan's conformal equivalent of the (affine) Levi-Civita connection.

Let $X$ be a manifold equipped with a conformal structure. This should be thought of as a distinguished line subbundle, $L^{* 2}$ of $\bigodot^{2} \Omega_{X}$, the sym-

Received September 29, 1987 and, in revised form, April 25, 1989. 
metric square of the cotangent bundle of $X$. Then sections of $L^{* 2}$ are metrics on $X$ differing only in overall scale. Such sections constitute the conformal class of metrics on $X$. The question of the existence of canonically defined differential invariants of this structure is a local one. So assume that a square root, $L$, of the dual $L^{2}$ exists and is fixed. Powers $L^{k}$ of $L$ are defined as usual, setting $L^{k}=\left(L^{*}\right)^{-k}$ if $k<0$. Sections of $L^{k}$ are called conformal weight $k$ functions. They are given locally by specifying a choice of metric $g$ in the conformal class and a function $\varphi$. When $g$ is rescaled to $\Omega^{2} g$, then $\varphi$ is replaced by $\Omega^{k} \varphi$.

The best known differential invariant of the conformal structure on $X$, of even dimension $2 n \geq 4$, is the conformally invariant Laplacian,

$$
\square: L^{-n+1} \rightarrow L^{-n-1} .
$$

Choose a metric in the conformal class, let $\nabla_{a}$ be its Levi-Civita connection, and let $R$ be its scalar curvature. Then

$$
\square=\nabla^{a} \nabla_{a}+\frac{n-1}{2(2 n-1)} R
$$

depends only on the conformal class-a different choice of scale for the metric alters the Levi-Civita connection and the scalar curvature in precisely opposite ways so that $\square$ is unchanged. This cancellation depends essentially on the fact that $\square$ is applied to sections of $L^{-n+1}$; it fails on any other power of $L$ and in particular on functions. It is rather remarkable, therefore, that, in four dimensions, the operator

$$
\square^{2}: L^{0} \rightarrow L^{-4}
$$

on functions given by

$$
f \rightarrow \nabla_{a}\left(\nabla^{a} \nabla^{b}+4 \Phi^{a b}+4 g^{a b} \Lambda\right) \nabla_{b} f
$$

is conformally invariant [18], [37]. Here, $\Phi^{a b}$ is (half) the tracefree Ricci tensor and $\Lambda=R / 24$. The direct verification of this fact relies on some quite remarkable cancellations and strongly suggests that some underlying principle is at work. (Indeed, once this is understood (Theorem 5.1.1), it will follow that on a conformal manifold of dimension $2 n$ there is a conformally invariant operator $\square^{p}: L^{p-n} \rightarrow L^{-p-n}$ whose highest order term is $\left(\nabla^{a} \nabla_{a}\right)$ for $1 \leq p \leq r$.

Eastwood and Singer discovered $\square^{2}$ after finding that $\left(\nabla^{a} \nabla_{a}\right)^{2}$ was conformally invariant in the flat case which followed from certain considerations in the twistor theory of R. Penrose [32]. This led Eastwood 
and Rice [17] to investigate the question of conformally invariant differential operators on Minkowski space more closely, characterizing them in terms of homomorphisms of (generalized) Verma modules (see also [26]). Such homomorphisms have been studied in considerable detail, recently [9], [28], so that this characterization determines all such operators. Moreover, the characterization determines all differential operators between homogeneous sheaves on compact homogeneous complex manifolds which are invariant under homogeneous translations.

The characterization follows from a study of the local geometry of a principal fiber bundle

$$
G \rightarrow G / P
$$

where $G$ is a complex semisimple Lie group and $P$ is a parabolic subgroup. For the purposes of this paper, the reader may take $G$ to be the conformal group $\mathrm{SO}(m+2, \mathrm{C})$ of the $m$-dimensional complex sphere and $P$ to be the conformal Poincare subgroup (or coverings of these). This identifies the formal jet bundle of a homogeneous vector bundle as a homogeneous vector bundle induced by the algebraic dual of a Verma module. Since linear differential operators are defined as linear maps from a jet bundle to the image bundle, and since invariant operators are determined by their action on the fiber of a jet bundle over a base point, it follows that invariant operators between sections of homogeneous vector bundles correspond to homomorphisms of Verma modules. The crucial point in the characterization is the identification of the differential operators on $G$ at the identity with the universal enveloping algebra $\mathscr{U}(\mathbf{g})$ of the Lie algebra $\mathbf{g}$ of $G$.

In the curved case, the group of conformal motions, $G$, is lost. Nonetheless, in dimensions three or more, there is a uniquely defined $P$-principle bundle

$$
\mathscr{G} \rightarrow \mathscr{G} / P=X
$$

and a g-valued one-form $\omega$, the normal Cartan connection, which is an isomorphism of vector spaces

$$
\Theta_{g} \mathscr{G} \rightarrow \mathbf{g} \quad \forall g \in \mathscr{G} .
$$

When $X$ is flat, $\mathscr{G}=G$ and $\omega$ is the Maurer-Cartan form identifying $\mathbf{g}$ with left invariant vector fields on $G$. The properties of $\omega$ and Verma modules in general turn out such that most homomorphisms of Verma modules induce differential invariants of the conformal structure, even though this is curved (Theorem 5.1.1).

The Cartan connection $\omega$ is constructed by suitably modifying the affine Levi-Civita connection of any metric in the conformal class [29]. 
This modification, which is the source of lower order "curvature correction terms", involves Ricci curvature so that only Ricci curvature and its derivatives can appear in such terms. It follows that most translation invariant linear differential operators on sections of a homogeneous vector bundle over a conformally flat manifold give rise to differential invariants of a curved conformal structure which may be constructed from the Levi-Civita connection and Ricci curvature of any metric in the conformal class.

In an earlier version of this paper it was thought that invariant curved analogues of powers $p>r$ of the Laplacian in $\operatorname{dim} 2 n$ existed. C. R. Graham has shown that the operator $\square^{3}: L \rightarrow L^{-s}$ in four dimensions has no analogue; I am very grateful to him for drawing my attention to this. The error lies in that part of 4.7.1 concerned with such operators. These, and others obtained from them, are the only flat operators which do not appear to admit curved analogues.

More important than the precise form of such invariants is the fact that they are constructed from a systematic theory which is based on the structure theory of Verma modules. This theory is now well understood and immediately suggests further results. For instance, homomorphisms of Verma modules occur in certain exact sequences, due to Bernstein, Gelfand, and Gelfand [7], [28]. The exactness of such sequences is dependent on the algebra structure of $\mathscr{U}(\mathbf{g})$. This is not available in the curved case; the obstruction is the extent to which

$$
\omega^{-1}: \mathbf{g} \rightarrow \Gamma\left(\mathscr{G}, \Theta_{\mathscr{G}}\right)
$$

is not a homomorphism of Lie algebras, which is determined by the conformal curvature. Indeed, the Bernstein-Gelfand-Gelfand sequence on a curved manifold is not even a complex, so that concatenation of differential invariants yields yet more invariants, which now involve Weyl curvature. The Weyl tensor itself is obtained in this way. A little careful study of the structure of the jet of the Weyl tensor using a particular Verma module shows that the Weyl tensor in dimension $2 n \geq 4$ is the subject of a $(2 n-2)$-order invariant differential operator. The result is a tracefree, symmetric, covariant rank-two tensor $B_{a b}$ of conformal weight $2-2 n$ which is conformally invariant, generalizing the Bach tensor in four dimensions.

It will be convenient but by no means necessary to work in the complex category throughout. The results obtained immediately generalize to the real situation (where some interesting new phenomena occur, because there fractional conformal weights are allowed [15]). 
The techniques of this paper are not confined to the conformal situation. Using [29] they extend to projective structures. They will also extend to structures locally modelled on any Hermitian symmetric space [4].

I should like to thank Michael Eastwood, Robin Graham and Ed Dunne for their encouragement and many useful conversations.

\section{Invariant linear differential operators on homogeneous spaces}

This section is devoted to the algebraic construction of differential operators on a flat conformal manifold which are invariant under conformal translations. Any flat conformal manifold in dimension at least three is locally isomorphic to a sphere, with its usual conformal structure; in this case, the conformal motions are readily checked to be the Lie group $\mathrm{SO}(m+2, \mathbf{C})$, and the stabilizer of any point is a subgroup isomorphic to the conformal Poincaré group $P=\left(\mathbf{S O}(m, \mathbf{C}) \times \mathbf{C}^{\times}\right) \ltimes \mathbf{C}^{m}$ realizing $\mathbf{C S}^{m}=G / P$ as a complex homogeneous space. The differential operators of interest act between sections of homogeneous vector bundles on this and intertwine the left translation action of $G$. The first step is to characterize such operators as adjoints of homomorphisms of (generalized) Verma modules for the conformal Lie algebra, so $(m+2, \mathbf{C})$, and the second is to use this characterization to classify them. The appendix contains a brief review of the basic representation theory which is used.

2.1. Differential operators. To begin with, recall the invariant definition of differential operators acting between sections of vector bundles over a complex manifold $X$. This is most easily stated using the language of locally free sheaves over the sheaf $\mathscr{O}_{X}$ of holomorphic functions on $X$, that is, in terms of sheaves of holomorphic sections of vector bundles. So let $\mathscr{F}$ denote the sheaf of holomorphic sections of a vector bundle $\mathscr{F}$ over a manifold $X$. Let $\mathscr{F}_{x}$ be the stalk of $\mathscr{F}$ at $x \in X$, i.e., the set of germs of holomorphic sections of $\mathscr{F}$ near $x$. Set

$$
\mathscr{F}_{x}^{r}(F)=\text { set of germs in } \mathscr{F}_{x} \text { vanishing to order } r \text { at } x .
$$

Then the $r$ th Jet bundle of $\mathscr{F}$ is defined by specifying its fiber at $x$ to be

$$
J_{x}^{r}(F)=\mathscr{F}_{x} / \mathscr{I}_{x}^{r+1}(\mathscr{F}) .
$$

Let $\mathscr{I}^{r}(F)$ be the associated locally free sheaf. The quotient mapping induces a homomorphism of $\mathbf{C}$-sheaves

$$
\mathscr{F} \stackrel{d^{r}}{\longrightarrow} \mathscr{F}^{r}(F),
$$

which is evidently a universal $r$ th-order differential. 
Taking a projective limit under the natural surjections $J^{r}(\underline{\mathscr{F}}) \rightarrow J^{r-1}(\underline{\mathscr{F}})$ gives the formal jet bundle

$$
J^{\infty}(F)=\lim ^{r} J^{r}(F)
$$

whose associated locally free sheaf is

$$
\mathscr{J}^{\infty}(F)=\lim \mathscr{I}^{r}(F) .
$$

Again, there is a universal differential operator

$$
d^{\infty}: \mathscr{F} \rightarrow \mathscr{I}^{\infty}(F)
$$

which gives the following definition [33].

Definition 2.2.1. A linear differential operator $\mathbf{D}: \mathscr{F} \rightarrow \mathscr{F}^{\prime}$ between locally free sheaves on $X$ is a sheaf homomorphism $\widetilde{\mathbf{D}} \circ d^{\infty}$ where $\widetilde{\mathbf{D}}: \mathscr{J}^{\infty}(F)$ $\rightarrow \mathscr{F}^{\prime}$ is a homomorphism of locally free sheaves which factors through $\mathscr{I}^{r} \mathscr{F}$ for some finite $r$. The order of $\mathbf{D}$ on a connected component of $X$ is the least such $r$.

Remark 2.1.2 If $\bigodot^{r} \Omega_{X}^{1}$ denotes the $r$ th symmetric power of the sheaf of holomorphic one-forms on $X$, then there are short exact sequences, the jet exact sequences [33]

$$
0 \rightarrow \bigodot^{r} \Omega_{X}^{1} \otimes \mathscr{F} \rightarrow \mathscr{I}^{r}(F) \stackrel{\varphi^{r}}{\longrightarrow} \mathscr{I}^{r-1}(F) \rightarrow 0
$$

If $\mathbf{D}$ is of order $r$, then the composition

$$
\bigodot^{r} \Omega_{X}^{1} \rightarrow \mathscr{I}^{r}(\mathscr{F}) \rightarrow \mathscr{F}^{\prime}
$$

is the symbol $\sigma_{\mathbf{D}}$ of $\mathbf{D}$.

Such technology is to be applied to vector bundles induced from a $P$ principal bundle $\mathscr{G} \rightarrow X$ whose structure group $P$ is a Lie group. These are constructed by specifying a representation $\pi: P \rightarrow \operatorname{End}(F)$ and setting $\underline{\mathscr{F}}=G \times{ }_{P} F$. Sections of $\underline{\mathscr{F}}$ are $F$-valued functions $f$ on $G$ satisfying

$$
f(g p)=\pi\left(p^{-1}\right) f(g) .
$$

This has a useful differential form; for each $u \in \mathbf{p}$, the Lie algebra of $P$, there is a natural realization of $u$ as a fiberwise vector field on $\mathscr{G} \rightarrow X$. Call this $u$ also. Then (2) implies

$$
u f=-d \pi(u) \cdot f \text {. }
$$

2.2. Characterization of invariant operators on homogeneous spaces. Restrict the discussion of the previous section by supposing that $\mathscr{G}=G$, a simply connected, semisimple, complex Lie group, and let $P$ be a (simply 
connected) parabolic subgroup. Then the quotient $X=G / P$ is a complex manifold which is compact, simply connected, Kähler, and projective. Its points correspond to subalgebras of $\mathbf{g}$, the Lie algebra of $G$, conjugate to p. Induced vector bundles on $X$ are usually referred to as homogenous vector bundles. Since right and left translations on $G$ commute, it is clear that the left translation action of $G$ on $X=G / P$ lifts to an action of $G$ on sections of a homogeneous vector bundle by

$$
L_{g}^{*} f\left(g^{\prime}\right)=f\left(g^{-1} g^{\prime}\right)
$$

for $g, g^{\prime} \in G$.

Definition 2.2.1. A translation invariant linear differential operator $\mathbf{D}$ between homogeneous sheaves over $X=G / P$ is a linear differential operator satisfying $\mathbf{D} \circ L_{g}^{*}=L_{g}^{*} \circ \mathbf{D}$.

Remark 2.2.2. Equation (4), differentiated, implies that the sheaf of sections of a homogeneous vector bundle is a sheaf of left $\mathscr{U}(\mathbf{g})$-modules. To say that a differential operator is invariant is to say that it intertwines this structure.

Evidently, such an operator is determined by its action on the fiber of $J^{\infty}(F)$ at a fixed base-point, say the identity coset $e P$. The first step in characterizing invariant differential operators is to identify this stalk. To do this, consider differential operators on $G$ sending $F$-valued functions on $G$ to functions on $G$. If the universal enveloping algebra $\mathscr{U}(\mathbf{g})$ is identified with left invariant differential operators on $G$, then the desired operators have the form

$$
f \rightarrow\left\langle f^{*}, u f\right\rangle
$$

for $f^{*}$ an $F^{*}$-valued function on $G, u \in \mathscr{U}(\mathbf{g})$, and $\langle$,$\rangle the natural$ pairing. When $f$ is a section of $\mathscr{F}$ and $u \in \mathbf{p},(3)$ implies

$$
\left\langle f^{*}, u f\right\rangle=\left\langle f^{*},-d \pi(u) \cdot f\right\rangle=\left\langle d \pi^{*}(u) \cdot f^{*}, f\right\rangle,
$$

where $\pi^{*}$ is the contragredient representation of $\pi$. Identifying the germ of the operator in (5) at the identity with an element of $\mathscr{U}(\mathbf{g}) \otimes F^{*}$ observe that $u \otimes f^{*}$ and $1 \otimes d \pi^{*}(u) \cdot f^{*}$ agree on sections of $F$. Let $I$ be the left $\mathscr{U}(\mathbf{g})$-submodule of $\mathscr{U}(\mathbf{g}) \otimes F^{*}$ generated by elements of the form

$$
u \otimes f^{*}-1 \otimes d \pi^{*}(u) \cdot f^{*}
$$

for $u \in \mathscr{U}(\mathbf{p})$. Then

$$
M_{\mathbf{p}}\left(F^{*}\right)=\left(\mathscr{U}(\mathbf{g}) \otimes F^{*}\right) / I=\mathscr{U}(\mathbf{g}) \otimes \mathscr{U}_{(\mathbf{p})} f^{*}
$$


is the space of differential operators from $\mathscr{F}$ to $\mathscr{O}$ at the identity coset, i.e.,

$$
M_{\mathbf{p}}\left(F^{*}\right)=\underset{\lim _{\longrightarrow}}{\longrightarrow} \operatorname{Hom}_{\mathbf{C}}\left(J^{r}(F)_{e P}, \mathbf{C}\right) .
$$

$M_{\mathrm{p}}\left(F^{*}\right)$ is a left $\mathrm{g}$-module by the left multiplication action of $\mathbf{g}$ on $\mathscr{U}(\mathbf{g})$. It is the g-module induced from the p-module $F^{*}$. By the PoincaréBirkhoff-Witt theorem, if $\mathbf{g}=\mathbf{p} \oplus \mathbf{u}_{-}$, then

$$
M_{\mathbf{p}}\left(F^{*}\right) \cong \mathscr{U}\left(\mathbf{u}_{-}\right) \otimes_{\mathbf{C}} F^{*}
$$

as a module for a reductive Levi factor $\mathbf{l}$ of $\mathbf{p}$ (which acts by the tensor product action deduced from its action on $\mathbf{u}_{-}$) and for $\mathbf{u}_{-}$, acting by left multiplication on the left factor. In the conformal case, $\mathbf{u}_{-}$is abelian and $\mathscr{U}\left(\mathbf{u}_{-}\right)$is the symmetric algebra on $\mathbf{u}_{-}$.

If $F^{*}$ is an irreducible finite-dimensional representation of $P$, then $M_{\mathrm{p}}\left(F^{*}\right)$ is a generalized Verma module [28]. By the general theory of irreducible representation of $\mathbf{p}$ (see the appendix), $F^{*}$ is generated by a single highest weight space, $F_{\lambda}^{*}$, and the decomposition (7) shows that, for any $f^{*} \in F_{\lambda}^{*}, 1 \otimes f^{*}$ generates $M_{\mathrm{p}}\left(F^{*}\right)$. Accordingly, denote it by $M_{\mathbf{p}}(\lambda)$ in the sequel. If $\mathbf{h}$ is a Cartan subalgebra of $\mathbf{g}$ (contained in $\mathbf{p}$ ), then the decomposition shows also that a Verma module is a direct sum of weight spaces. The generating weight space is distinguished by the fact that its weight is highest amongst all nontrivial weights for the Verma module (in the usual partial order on weights determined by the choice of a Borel subalgebra contained in $\mathbf{p}$ ). There exist weight spaces in a Verma module which are maximal but not highest-these are most significant, as will be seen.

The grading on $\mathscr{U}(\mathbf{g})$ induces a filtration on $M_{\mathrm{p}}\left(F^{*}\right)$, which is exactly that at (6). This identifies

$$
J_{e P}^{\infty}(F)=\lim _{\mathbf{L}} \operatorname{Hom}_{\mathbf{C}}\left(M_{\mathbf{p}}^{r}\left(F^{*}\right), \mathbf{C}\right)=\left(M_{\mathbf{p}}\left(F^{*}\right)\right)^{*} .
$$

The identification at (6) depends on working at the identity of $G$. Under the right action of $P$ on $G$ this varies by the adjoint action of $P$. Therefore, the sheaf of differential operators from $\mathscr{F}$ to $\mathscr{O}$ is induced by $M_{\mathbf{p}}\left(F^{*}\right)$. Similarly, the jet sheaf $\mathscr{J}^{\infty}(F)$ is induced by $\left(M_{\mathbf{p}}\left(F^{*}\right)\right)^{*}$ with the evident projective limit structure. The associated composition series induces the jet exact sequences (1).

It follows that an invariant differential operator D: $\mathscr{F} \rightarrow \mathscr{F}^{\prime}$ between homogeneous sheaves induced by representations $F, F^{\prime}$ of $P$ corresponds contragrediently to a p-module homomorphism

$$
\mathbf{D}^{*}: F^{*} \rightarrow M_{\mathbf{p}}\left(F^{*}\right) \text {. }
$$


Now restrict $F, F^{\prime}$ to be irreducible and let $1 \otimes v$ and $1 \otimes v^{\prime}$ be maximal highest weight generating vectors of $M_{\mathrm{p}}(\mu)=M_{\mathrm{p}}\left(F^{*}\right)$ and $M_{\mathrm{p}}\left(\mu^{\prime}\right)=$ $M_{\mathbf{p}}\left(F^{\prime *}\right)$. Then $w=\mathbf{D}^{*} 1 \otimes v^{\prime}$ is maximal and of weight $\mu^{\prime}$ in $M_{\mathbf{p}}(\mu)$. Any such $w$, on the other hand, generates a copy of $F^{\prime *}$ in $M_{\mathrm{p}}(\mu)$ under the action of $\mathscr{U}(\mathbf{p})$ and so determines a $\mathbf{D}^{*}$, hence an invariant differential operator. Finally, under the action of $\mathscr{U}(\mathbf{g}), w$ generates a copy of $M_{\mathbf{p}}\left(F^{\prime *}\right)$ in $M_{\mathbf{p}}(\mu)$ and all such copies arise this way (see [17, 26]).

Theorem 2.2.3. The translation invariant linear differential operators between irreducible homogeneous sheaves over a complex homogeneous variety $X=G / P$ are characterized by homomorphisms of Verma modules for $\mathbf{g}$ induced from irreducible representations of $\mathbf{p}$.

2.3. An example on $\mathbf{C P}^{1}$. Let $G=\mathrm{SL}(2, \mathbf{C})$ and $P=B=$ upper triangular matrices. Then $G$ acts transitively on $\mathbf{C} \mathbf{P}^{1}$ and the stabilizer of a point is conjugate to $B$. So $X=G / B=\mathbf{C P}^{1}$. The irreducible homogeneous bundles on $X$ are all line bundles (since the irreducible representations of the Borel group $B$ are all one-dimensional). They may be identified with powers of the tautological bundle; obtain bundles $L(n)$ for integral $n$, induced by the representation of $B$ on $\mathbf{C}$ in which $\left(\begin{array}{cc}z & 0 \\ 0 & z^{-1}\end{array}\right)$ acts by multiplication by $z^{-n}$ and $\left(\begin{array}{ll}1 & z \\ 0 & 1\end{array}\right)$ acts as the identity. Denote the sheaves of holomorphic sections of these bundles by $\mathscr{O}(n)$. The Lie algebra $\mathbf{g}=\mathbf{s l}(2, \mathbf{C})$ is generated by

$$
x=\left(\begin{array}{ll}
0 & 1 \\
0 & 0
\end{array}\right), \quad y=\left(\begin{array}{ll}
0 & 0 \\
1 & 0
\end{array}\right), \quad h=\left(\begin{array}{cc}
1 & 0 \\
0 & -1
\end{array}\right)
$$

which satisfy

$$
[x, y]=h, \quad[h, x]=2 x, \quad[h, y]=-2 y ;
$$

all other commutators are zero. b may be taken to be the span of $x, h$ whilst $h$ spans a Cartan subalgebra of $\mathbf{g}$. The Verma module whose algebraic dual is the stalk at the identity coset of the formal jet bundle of $L(n)$ is then

$$
M(n)=\mathbf{C}[y] \otimes_{\mathbf{C}} \mathbf{C}(n),
$$

where $\mathbf{C}(n)$ is a copy of $\mathbf{C}$ on which $h$ acts by multiplication by $n$ and $x$ trivially. A highest weight vector in $M(n)$ has the form $1 \otimes v$ for nonzero $v \in \mathbf{C}(n)$. 
Let $\alpha$ and $\beta$ be highest weight vectors in $M(n-1)$ and $M(-n-1)$, respectively. Then calculate

$$
\begin{aligned}
x y^{n} \alpha & =\left\{[x, y] y^{n-1}+y\left(x y^{n-2}\right)\right\} \alpha \\
& =h y^{n-1} \alpha+y h y^{n-2} \alpha+\cdots+y^{n-1} h \alpha \\
& =\{(1-n)+(3-n)+\cdots+(n-1)\} \alpha=0
\end{aligned}
$$

and

$$
h y^{n} \alpha=(-n-1) y^{n} \alpha
$$

so that $y^{n} \alpha$ is maximal in $M(n-1)$ of weight $-n-1$. Thus the assignment $\beta \mapsto y^{n} \alpha$ yields a homomorphism of Verma modules $M(-n-1) \rightarrow$ $M(n-1)$ and a corresponding $n$th order invariant differential operator

$$
\partial^{n}: \mathscr{O}(n-1) \rightarrow \mathscr{O}(-n-1) .
$$

These are the conformally invariant powers of edth described in [19]. They may be determined as follows: let $\left[\pi^{A}\right]$ be homogeneous coordinates on $\mathbf{C P} \mathbf{P}^{1}$ and represent a section of $\mathscr{O}(n)$ by a function $f$ on $\mathbf{C}^{2}$, homogeneous of degree $n$. Then

$$
\pi^{A_{1}} \pi^{A_{2}} \cdots \pi^{A_{n}} \partial^{n} f=\frac{\partial}{\partial \pi^{A_{1}}} \frac{\partial}{\partial \pi^{A_{2}}} \cdots \frac{\partial}{\partial \pi^{A_{n}}} f .
$$

2.4. Symbols of invariant operators. Any translation invariant differential operator $\mathbf{D}: \mathscr{F} \rightarrow \mathscr{F}^{\prime}$ has a symbol $\sigma_{\mathbf{D}}: \bigodot^{r} \Omega_{X}^{1} \otimes \mathscr{F} \rightarrow \mathscr{F}^{\prime}$. By Schur's lemma, since $\Omega_{X}^{1}$ is a homogeneous vector bundle, this corresponds to a projection under the decomposition of $\bigodot^{r} \mathbf{u}_{-} \otimes F$ into a direct sum of irreducibles for 1 . This fact, which might jocularly be called a symbol principle, is very useful. On the one hand, it often eliminates several possibilities; on the other hand, if a possible symbol exists between irreducible homogeneous sheaves $\mathscr{O}_{\mathbf{p}}(\lambda)$ and $\mathscr{O}_{\mathbf{p}}(\mu)$, then a vector of weight $\mu$ in $M_{\mathbf{p}}(\lambda)$ is determined by the symbol, using the usual Clebsch-Gordon decomposition formulas. It is automatically maximal for $\mathbf{l}$, and it remains to check that it is annihilated by $\mathbf{u}$. In the conformal case, $\mathbf{u}$ is irreducible as an $\mathbf{l}$ module, and so there is only one condition to check.

\section{The four-dimensional case}

The next example, following [17], is the physically most interesting case originally studied using the twistorial techniques of $\mathrm{R}$. Penrose. This concerns the complexified four-sphere, sometimes referred to as complexified, compactified Minkowski space. It will form the basis of explicit calculations in the curved situation later on in this paper and so it will be treated 
in some detail. The group of conformal motions of the complex foursphere is $\mathrm{SO}(6, \mathrm{C})$ and the stabilizer of a point is the conformal Poincare group, $\left\{\mathrm{SO}(4, \mathrm{C}) \times \mathrm{C}^{\times}\right\} \times \mathbf{C}^{4}$. As remarked above, any conformally flat complex four-manifold is locally conformal to this conformal manifold. Let $G$ be the simply connected covering of $\mathrm{SO}(6, \mathrm{C}), P$ be the corresponding simply connected stabilizer, and $X=G / P$. Then $G=\operatorname{SL}(4, C)$ and $P$ consists of the subgroup of matrices of the form

$$
\left\{\begin{array}{llll}
* & * & * & * \\
* & * & * & * \\
0 & 0 & * & * \\
0 & 0 & * & *
\end{array}\right\}
$$

which realizes $X=\mathbf{G r}_{2}\left(\mathbf{C}^{4}\right)$ as the Klein quadric.

3.1. Notation. Fix generators of the Lie algebra $\mathrm{gl}(4, \mathrm{C})$ by writing a symbol from the following matrix for the generator which has a one in the corresponding position and zeros elsewhere:

$$
\left(\begin{array}{llll}
H^{1} & X_{1} & x_{2} & x_{4} \\
Y_{1} & H_{2} & x_{1} & x_{3} \\
y_{2} & y_{1} & H_{3} & X_{2} \\
y_{4} & y_{3} & Y_{2} & H_{4}
\end{array}\right) .
$$

The off diagonal generators together with $h_{i}=H_{i}-H_{i+1}$ for $i=1,2,3$ form generators of $\mathbf{g}=\mathbf{s l}(4, \mathbf{C})$. Let $\mathbf{h}=\operatorname{span}\left\{h_{i}\right\}, \mathbf{n}=\operatorname{span}\left\{X_{i}, x_{i}\right\}$, $\mathbf{u}=\operatorname{span}\left\{x_{i}\right\}, \mathbf{l}=\oplus \operatorname{span}\left\{X_{i}, Y_{i}\right\}, \mathbf{u}_{-}=\operatorname{span}\left\{y_{i}\right\}, \mathbf{b}=\mathbf{h} \oplus \mathbf{n}$, and $\mathbf{p}=\mathbf{l} \oplus \mathbf{u}$. Then $\mathbf{h}$ is a Cartan subalgebra of $\mathbf{g}, \mathbf{n}$ is a choice of positive roots spaces, and $\mathbf{p}$ is the parabolic subalgebra corresponding to $P$, with a fixed Levi decomposition. If $\alpha_{1} \alpha_{2} \alpha_{3}$ indicates the nodes associated to the simple roots of $\mathbf{g}$, then the simple roots spaces are

$$
\mathbf{g}_{\alpha_{1}}=\mathbf{C} \cdot X_{1}, \quad \mathbf{g}_{\alpha_{2}}=\mathbf{C} \cdot x_{1}, \quad \mathbf{g}_{\alpha_{3}}=\mathbf{C} \cdot X_{2} .
$$

Recall that $\left[h_{i} v\right]=\left\langle\lambda, \alpha_{i}^{\vee}\right\rangle v$ for $v \in \mathbf{g}_{\lambda}$. Clearly, $\mathbf{p}=\bullet \longleftrightarrow$, and $M_{\mathbf{p}}(\lambda) \cong \mathbf{C}[\mathbf{u}] \otimes_{\mathbf{C}} F_{\mathbf{p}}(\lambda)$ (for the notation see the appendix).

3.2. Homogeneous bundles. Irreducible homogeneous vector bundles on $X$ are specified by symbols of the form $\stackrel{p q r}{\sim}$, for integers $p, q, r$ with $p, r \geq 0$. The holomorphic cotangent bundle $\Omega_{X}^{1}$ of $X$ is a homogeneous bundle corresponding to the representations of $\mathbf{p}$ on its nilpotent Levi 
factor $\mathbf{u} ; \mathbf{u}$ is irreducible and

$$
\begin{aligned}
& \Omega_{X}^{1}=\stackrel{1-21}{\longrightarrow \sim},
\end{aligned}
$$

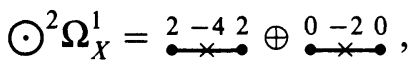

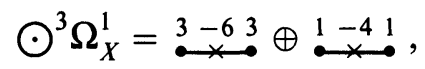

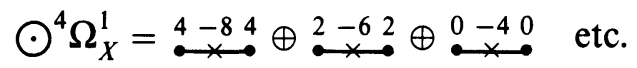

whilst

$$
\begin{aligned}
& \Omega_{X}^{2}=\stackrel{2-30}{\sim} \oplus \stackrel{0-32}{\longrightarrow \sim}, \\
& \Omega_{X}^{3}=\stackrel{1-41}{\stackrel{*}{\longrightarrow},}, \\
& \Omega_{X}^{4}=\stackrel{0-40}{\longrightarrow} \text {. }
\end{aligned}
$$

The sheaf of conformal weight $n$ functions, which scale by $f \rightarrow \Omega^{n} f$ under a rescaling $g \rightarrow \Omega^{2} g$ of a local choice of metric within the conformal class, is

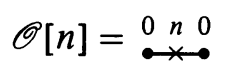

and the spinor sheaves are

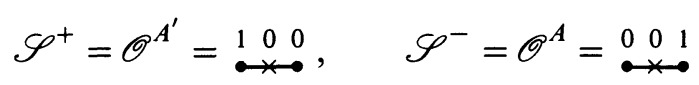

following the abstract index notation of [31]. Indices may be "lowered" at the expense of acquiring a conformal factor:

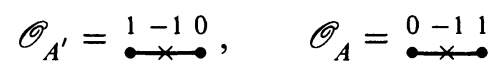

and generally

$$
\mathscr{O}^{\left(A_{1}^{\prime} \cdots A_{p}^{\prime}\right)\left(A_{1} \cdots A_{r}\right)}[q]=\stackrel{p \underset{*}{q} r}{ } r .
$$

3.3. Invariant operators on forms. First attempt to compute the invariant differential operators from functions to forms. (Functions are sections of the trivial homogeneous bundle $\begin{array}{lll}0 & 0 & 0 \\ 0 & -0 & 0\end{array}$.) Using symbols from (2.4) and (9), (10), the only possibilities for invariant differential operators are the following:

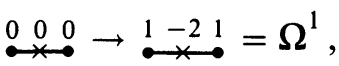

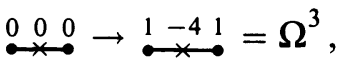

$$
\begin{aligned}
& \stackrel{0 \underset{0}{0} 0}{0} \rightarrow \stackrel{0-40}{\stackrel{0}{\longrightarrow}}=\Omega^{4} \text {. }
\end{aligned}
$$

Let $\alpha \in M_{\mathbf{p}}\left(\begin{array}{ccc}0 & 0 & 0 \\ 0 \rightarrow 0\end{array}\right)$ be a highest weight vector. Clebsch-Gordon decomposition yields vectors

$$
y_{1} \alpha, \quad y_{1}\left(y_{1} y_{4}-y_{2} y_{3}\right) \alpha, \quad\left(y_{1} y_{4}+y_{4} y_{1}-y_{2} y_{3}-y_{3} y_{2}\right)^{2} \alpha
$$


for (11), (12), and (13), respectively. These are maximal for 1 (i.e., annihilated by $X_{i}$ ) and so yield homomorphisms of Verma modules as $\mathscr{U}(\mathbf{l})$ modules. To promote these to $\mathscr{U}(\mathbf{g})$-module homomorphisms requires that these vectors be annihilated by $x_{1}$, hence by all $x_{i} ;(11)$ and (13) are whilst (12) is not. It follows that there are translation invariant differential operators

$$
d: \mathscr{O} \rightarrow \Omega_{X}^{1} \quad \text { (the usual exterior differential) }
$$

and

$$
\left.\square^{2}=(\nabla \cdot \nabla)^{2}: \mathscr{O} \rightarrow \Omega_{X}^{4} \quad \text { (for any flat Levi-Civita connection } \nabla\right) .
$$

It is easy [17] to check that the remaining invariant operators between forms are the usual exterior differentials together with the composition

$$
\Omega^{1} \stackrel{d^{+}}{\longrightarrow} \Omega_{+}^{2} \stackrel{d}{\longrightarrow} \Omega^{3} .
$$

For example, if $\beta$ is a highest weight vector of $M_{\mathbf{p}}\left(\begin{array}{ll}1-2 & 1 \\ \sim *\end{array}\right)$, then a maximal vector of weight $\underset{-x}{2-3} 0$ in $M_{\mathrm{p}}\left(\begin{array}{cc}1-2 & 1 \\ -*\end{array}\right)$, given by $\left(-y_{3}+y_{1} Y_{2}\right) \beta$, induces the operator $d^{+}$, whilst for $\gamma$ a highest weight vector of $M_{\mathbf{p}}\left(\begin{array}{lll}1 & -4 & 1 \\ 0 & - & -\end{array}\right)$ a maximal vector $\left(y_{1} Y_{1} Y_{2}-y_{3} Y_{1}+y_{1} Y_{2}-y_{4}\right) \gamma$ of weight $\begin{gathered}0-40 \\ \longrightarrow\end{gathered}$ in $M_{\mathrm{p}}\left(\begin{array}{ccc}1-4 & -4 \\ -\sim\end{array}\right)$ induces $d: \Omega^{3} \rightarrow \Omega^{4}$.

In summary, the following is an exhaustive list of invariant differential operators between forms on Minkowski space:

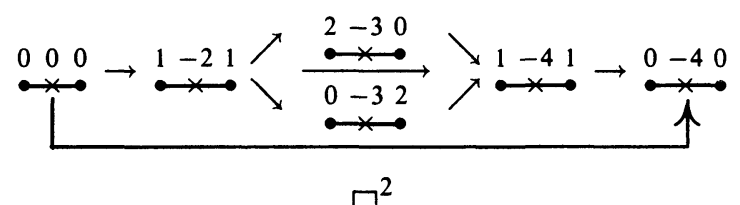

Indeed, it will be shown in $\S 4.2$ that any translation invariant differential operator on forms must be one of those in (14).

3.4. A third order operator on spinors. In the extension of these results to curved conformal structures it will be useful to give some nontrivial examples. One of these will be based on a third order operator $\begin{gathered}1-23 \\ \rightarrow x \rightarrow\end{gathered} \rightarrow$ $\stackrel{4-50}{4}$ given by

$$
\lambda_{(A B C)}^{A^{\prime}} \rightarrow \nabla_{\left(A^{\prime}\right.}^{A} \nabla_{B^{\prime}}^{B} \nabla_{C^{\prime}}^{C} \lambda_{\left.D^{\prime}\right) A B C}
$$

and induced by the following maximal vector:

$$
\left(-y_{3}+y_{1} Y_{2}\right)\left(-2 y_{3}+y_{1} Y_{2}\right)\left(-3 y_{3}+y_{1} Y_{2}\right) \alpha \text {. }
$$


3.5. Powers of the Laplacian. There is an infinite family of operators which are powers of the Laplacian $\nabla^{a} \nabla_{a}$, acting on conformally weighted functions:

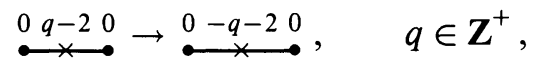

by $f \rightarrow\left(\nabla^{a} \nabla_{a}\right)^{q} f$ induced by the maximal vector

$$
\left(y_{1} y_{4}+y_{4} y_{1}-y_{2} y_{3}-y_{3} y_{2}\right)^{q} \alpha .
$$

Each of these will give rise to a differential invariant of a curved conformal structure in four dimensions.

\section{Homomorphisms of Verma modules}

Much is known about the existence of homomorphisms of generalized Verma modules. This section gives a brief review of the relevant aspects of the subject. (A brief review of the Weyl group $W_{\mathrm{g}}$ of a semisimple Lie algebra $\mathbf{g}$ and a subgraph $W^{\mathbf{p}}$ associated to a parabolic subalgebra $\mathbf{p}$ is given in the appendix.)

Any Verma module $M_{\mathrm{p}}(\lambda)$ has a unique maximal submodule; the quotient by this submodule, $L(\lambda)$, is independent of $\mathbf{p}$ and is the unique irreducible g-module generated by a highest weight space of weight $\lambda$. The unique maximal submodule is a sum (not usually direct) of $M_{\mathbf{p}}(\mu)$; it follows [28] that each $M_{\mathbf{p}}(\lambda)$ has Jordan-Hölder composition series whose subquotients have the form $L(\mu)$ for appropriate $\mu$.

4.1. Infinitesimal character. The first important fact is the notion of infinitesimal character. Since $M_{\mathbf{p}}(\lambda)$ is generated over $\mathscr{U}(\mathbf{g})$ by a single highest weight space, an element $z$ of the center $\mathscr{Z}(\mathbf{g})$ of $\mathscr{U}(\mathbf{g})$ must act by a scalar $\xi \lambda(z)$ determined by $\lambda . \xi_{\lambda} \in \mathscr{Z}(\mathbf{g})^{*}$ is called the infinitesimal character of $\lambda$. Clearly, a homomorphism $M_{\mathbf{p}}(\lambda) \rightarrow M_{\mathrm{p}}(\mu)$ can exist only if $\xi_{\lambda}=\xi_{\mu}$. It is a classical theorem of Harish-Chandra [23] that this is possible if and only if $\lambda+\rho$ and $\mu+\rho$ are conjugate under the action of the Weyl group $W_{\mathbf{g}}$ of $\mathbf{g}$. Here, $\rho=\frac{1}{2} \sum_{\alpha \in \Delta^{+}(\mathbf{g})} \alpha$ is represented in the weight notation of the appendix by inscribing a one over every node of the Dynkin diagram for $\mathbf{g}$. For $w \in W_{\mathbf{g}}$ and a weight $\lambda$, the affine action of $W_{\mathbf{g}}$ is defined by $w \cdot \lambda=w(\lambda+\rho)-\rho$.

Lemma 4.1.1. $A$ translation invariant linear differential operator $\mathbf{D}: \mathscr{O}_{\mathbf{p}}(\mu) \rightarrow \mathscr{O}_{\mathbf{p}}(\lambda)$ may exist only if $\mu=w \cdot \lambda$ for some $w \in W_{\mathbf{g}}$.

Example 4.1.2. In $\S 3$, the use of symbols suggests a possible invariant operator $\mathscr{O} \rightarrow \stackrel{2-42}{\longrightarrow}$ (for instance). It is easy to check, however, using the 
recipe for reflections in the appendix, that there does not exist an element

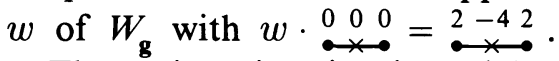

The easiest situation in which to apply the lemma is for $\lambda$ dominant for $\mathbf{g}$. Then $\lambda+\rho$ is nonsingular and any weight $\mu$ dominant for $\mathbf{p}$ with infinitesimal character $\xi_{\lambda}$ is said to have regular infinitesimal character and the bundle $\mathscr{O}_{\mathbf{p}}(\mu)$ is called regular. A complete list of such $\mu$ is given by

$$
\left\{w \cdot \lambda \mid w \in W^{\mathbf{p}}\right\},
$$

where $W^{\mathbf{p}}$ is the Hasse subgraph of $W_{\mathbf{g}}$ associated to the parabolic $\mathbf{p}$.

4.2. The general regular case in dimension four. Consider the case of four-dimensions, again, with $\lambda=\stackrel{p q}{q} r$, where $p, q, r \geq 0$ so that $\lambda$ is dominant for $\mathbf{g}$. Applying the elements of $W^{\mathbf{p}}$ to $\lambda$ and retaining the directed graph structure of $W^{\mathbf{p}}$ yields the following list of regular bundles with infinitesimal character $\xi_{\lambda}$ :

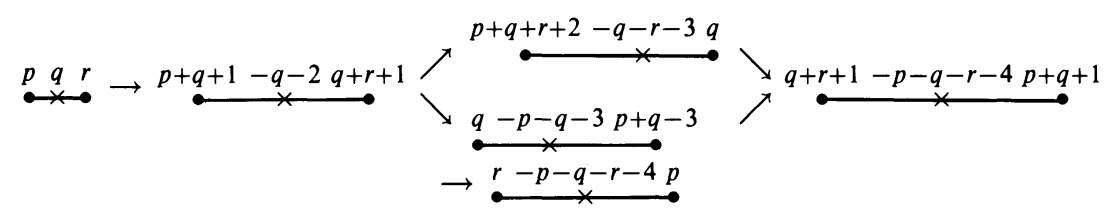

In particular, the only invariant operators on forms are those of (14), [17].

The arrows in this list indicate all the invariant differential operators between the bundles appearing in the list; this is a consequence of the earlier classification of the invariant operators on forms and the translation principle of Jantzen and Zuckerman [3], [17], [36].

4.3. The Borel case. The original study of Verma modules [35], [7] was made for $\mathbf{p}=\mathbf{b}$ a Borel subalgebra of $\mathbf{g}$; there is a complete classification of all homomorphisms between these modules, due to Verma and Bernstein, Gelfand, and Gelfand [7], [35]:

Theorem 4.3.1. The following are equivalent:

(i) There exists a translation invariant linear differential operator $\mathbf{D}$ : $\mathscr{O}_{\mathbf{b}}(\lambda) \rightarrow \mathscr{O}_{\mathbf{b}}(\mu)$ on $G / B$, unique up to scale.

(ii) There is a sequence $\left\{\beta_{i}\right\}$ of positive roots of $\mathbf{g}$ such that

(a) $\mu=\sigma_{\beta_{n}} \sigma_{\beta_{n-1}} \cdots \sigma_{1} \cdots \lambda$,

(b) $\left\langle\lambda+\rho, \beta_{1}^{\vee}\right\rangle \geq 0$,

(c) for each $p=1,2, \cdots, n,\left\langle\left(\sigma_{\beta_{p-1} \sigma_{\beta_{p-2}} \cdots \sigma_{\beta_{1}}} \cdot \lambda\right)+\rho, \beta^{\vee}\right\rangle \geq 0$.

(iii) $L(\mu)$ is a subquotient of $M_{\mathbf{b}}(\lambda)$. 
In the realm of regular infinitesimal character this becomes:

Corollary 4.3.2. Let $\lambda$ be a dominant weight for $\mathbf{g}$. There exists a translation invariant linear differential operator $\mathbf{D}: \mathscr{O}_{\mathbf{b}}(w \cdot \lambda) \rightarrow \mathscr{O}_{\mathbf{b}}\left(w^{\prime} \cdot \lambda\right)$ on $G / B$ if and only if $w \preccurlyeq w^{\prime}$ in the Weyl group $W_{\mathbf{g}}$ of $\mathbf{g}$.

Example 4.3.3. Consider $\lambda=$\begin{tabular}{cc}
$0-10$ \\
\multirow{x}{*}{. }
\end{tabular} . defining a line bundle on the complete flag manifold $F_{123}$ of $\mathbf{C}^{4}$. Labelling the simple roots of $\operatorname{sl}(4, \mathbf{C})$

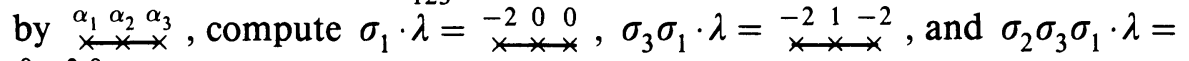
a -30 . It follows from the theorem that there is an invariant differential

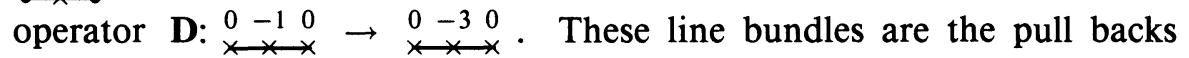
of the conformal line bundles $0-10$ and $0-30$ on Minkowski space. The homomorphism of induced modules is given by the maximal vector $y_{1}^{2} Y_{1} Y_{2} \alpha$ (where $\alpha$ is highest in $M_{\mathbf{b}}\left(\begin{array}{cc}0-1 & 0 \\ \hdashline\end{array}\right)$ ). Let $\nabla, \partial$, and $\partial^{\prime}$ be the relative differentials along the projections to the spaces of partial $(1,3)$, $(1,2)$, and $(2,3)$ flags. Then $\mathbf{D}=\nabla^{2} \partial \partial^{\prime}$.

4.4. The case of a general parabolic. Unfortunately, the parabolic subalgebras of interest in conformal geometry are far from being Borel and the theory of homomorphisms of generalized Verma modules (when $\mathbf{p}$ is not Borel) is far more complicated.

Certain homomorphisms, known as standard homomorphisms [28], may be deduced from the Borel case as follows. There is a natural fibration $G / B \stackrel{\tau}{\longrightarrow} G / P$ if $\mathbf{p}$ is chosen to contain $\mathbf{b}$. Then a section of $\mathscr{O}_{\mathbf{p}}(\lambda)$ on $G / P$ may be pulled back to a section of $\tau^{-1} \mathscr{O}_{\mathbf{p}}(\lambda)$ on $G / B$. This injects onto $\tau^{*} \mathscr{O}_{\mathbf{p}}(\lambda)$ which has a composition series by homogeneous line bundles, one for each weight of $F_{\mathbf{p}}(\lambda)^{*}$, taken with multiplicity. The highest of these is a single occurrence of $\mathscr{O}_{b}(\lambda)$. So obtain a section of this line bundle. This may be the subject of an invariant operator

$$
\mathbf{D}_{B}: \mathscr{O}_{\mathbf{b}}(\lambda) \rightarrow \mathscr{O}_{\mathbf{b}}(\mu)
$$

for some $\mu$ which is dominant for $\mathbf{p}$. This yields an invariant differential operator

$$
\mathbf{D}_{P}=\tau_{*} \circ \mathbf{D}_{B} \circ \tau^{-1}: \mathscr{O}_{\mathbf{p}}(\lambda) \rightarrow \mathscr{O}_{\mathbf{p}}(\mu)
$$

(An algebraic interpretation of this geometric construction is easily given.)

The difficulty is that this new invariant differential operator may well be zero- $\mathbf{D}_{B}$ may annihilate the image of $\tau^{-1} \mathscr{O}_{\mathbf{p}}(\lambda)$; Example 4.3 .3 is a case in point. Nonetheless, a nonzero invariant differential operator may still exist: in the example, this is provided by the Laplacian $\square: \stackrel{0-10 \rightarrow 0-30}{0} \rightarrow$ The corresponding homomorphism and this new operator are called nonstandard; $\mathbf{D}_{P}$ and its homomorphism are called standard [28]. 
Remark 4.4.1. (i) It is easy to determine when $\mathbf{D}_{P}=0$. In particular, if $\lambda$ is dominant, $\mu=w \cdot \lambda, \nu=w^{\prime} \cdot \lambda$ with $w^{\prime}=w \sigma_{\alpha}$, and $l\left(w^{\prime}\right)=l(w)+1$, then $\mathbf{D}_{p} \neq 0[3],[28]$.

(ii) If a nontrivial homomorphism $M_{\mathbf{p}}(\mu) \rightarrow M_{\mathbf{p}}(\lambda)$ exists then $L(\mu)$ is a composition factor of $M_{\mathbf{b}}(\lambda)$; so by Theorem 4.3.1, there is a nontrivial invariant differential operator $\mathscr{O}_{b}(\lambda) \rightarrow \mathscr{O}_{b}(\mu)$. The occurrence of nonstandard homomorphisms then corresponds to the multiple occurrence of $L(\mu)$ as a subquotient of $M_{\mathbf{b}}(\lambda)$. Determining these multiplicities is the subject of the Kahzdan-Lusztig conjecture [6], [11] and is an extremely subtle question.

(iii) Nonstandard invariant differential operators are not direct images of invariant operators on $G / B$; however, it seems that some, if not all, can be constructed by taking direct images of certain complexes of invariant operators on $G / B$. This construction is a form of the Penrose transform [5], [14], [16].

4.5. The regular conformal case. Boe and Collingwood [9] have determined all the homomorphisms of generalized Verma modules of regular infinitesimal character for $\mathbf{g}$ and $\mathbf{p}$ as in the conformal situation.

Recall (see appendix) that if $w \in W_{\mathbf{g}}$ then $w \cdot \lambda$ is dominant for $\mathbf{p}$ if and only if $w \in W^{\mathbf{p}}$, the Hasse subgraph of $W_{\mathbf{g}}$ associated to $\mathbf{p}$. In odd dimensions $2 n+1$, there is exactly one such $w$ with length $l$ for $0 \leq l \leq 2 n+1$. In even dimensions $2 n$, the same is true for $0 \leq l \leq n-1$ and $n+1 \leq l \leq 2 n$, with two possible $w$ 's of length $n$. Labelling the simple roots of $\mathbf{g}$ as in the appendix, let

$$
w^{0}=\sigma_{\alpha_{1}} \sigma_{\alpha_{2}} \cdots \sigma_{\alpha_{n-1}} \sigma_{\alpha_{n}} \sigma_{\alpha_{n-1}} \cdots \sigma_{\alpha_{2}} \sigma_{\alpha_{1}}
$$

be the longest element of $W^{\mathbf{p}}$ in even dimensions $2 n$. Then, in the language of invariant different operators, Boe and Collingwood have proved

Theorem 4.5.1. Let $\lambda$ be a dominant integral weight for $\mathbf{g}$. Then there is a nontrivial invariant differential operator

$$
\mathscr{O}_{\mathbf{p}}(w \cdot \lambda) \rightarrow \mathscr{O}_{\mathbf{p}}\left(w^{\prime} \cdot \lambda\right)
$$

if and only if $l(w)<l\left(w^{\prime}\right)$ and

(i) in odd dimensions, $l\left(w^{\prime}\right)=l(w)+1$,

(ii) in even dimensions, $l\left(w^{\prime}\right)=l(w)+1$ or $w^{\prime}=w^{0} w$, in which case $l\left(w^{\prime}\right)=2 n-l(w)$.

The differential operator is unique up to scale.

All of these operators are standard, except for the second possibility in (ii) with $l(w) \leq n-2$ which yields nonstandard operators. The operator 
in (ii) with $l(w)=n-1$ is either composition,

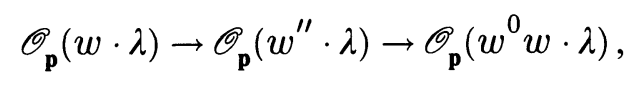

where $w^{\prime \prime}$ has length $n$. All other compositions are zero.

The theorem may be represented pictorially by letting • represent $\mathscr{O}_{\mathbf{p}}(w \cdot \lambda), l(w)$ increasing from left to right across the diagram, and letting arrows represent invariant differential operators.

The odd dimensional case:

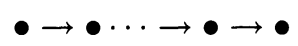

The even dimensional case:

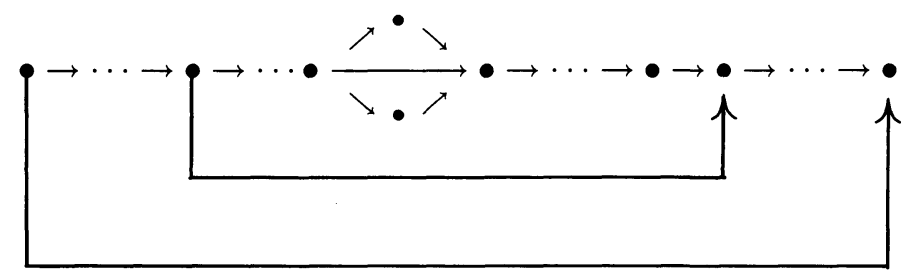

Only the long operators, corresponding to the longest arrow in this diagram in nontrivial regular integral infinitesimal character, will fail to admit curved analogues.

Example 4.5.2. If $\lambda$ is the zero weight, then $\mathscr{O}_{\mathbf{p}}(w \cdot \lambda)$ ranges over the irreducible components of the forms on $X$ (of degree $l(w)$ ). In odd dimensions, the only invariant differential operators between these are the usual exterior differentials. In even dimensions, the exterior differentials are augmented by the differentials

$$
\underbrace{d * d * \cdots * d * d}_{2(n-p) \text { factors of } d}: \Omega_{X}^{p} \rightarrow \Omega_{X}^{2 n-p}, \quad 0 \leq p \leq n-1,
$$

where $*$ is the Hodge star operator for a flat metric in the conformal class. In particular,

$$
\square^{2 n}=\left(\nabla^{a} \nabla_{a}\right)^{2 n}: \mathscr{O} \rightarrow \Omega_{X}^{2 n}
$$

( $\nabla_{a}$ a flat Levi-Civita connection) is conformally invariant.

Example 4.5.3. Let

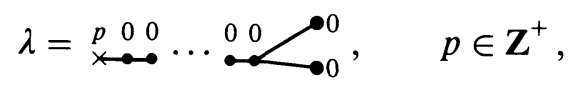

so that $\mathscr{O}_{\mathbf{p}}(\lambda)=L^{p}$ and $\mathscr{O}_{\mathbf{p}}\left(w^{0} \cdot \lambda\left(=L^{-p-2 n}\right.\right.$; deduce a conformally invariant power of the Laplacian:

$$
\square^{(p+n)}=\left(\nabla^{a} \nabla_{a}\right)^{(p+n)}: L^{p} \rightarrow L^{-p-2 n} .
$$


Remark that all of these operators will yield conformally invariant differential operators on an arbitrary conformal manifold, by virtue of Theorem 5.1.1.

4.6. The singular conformal case. Rather less is known about the possible homomorphisms between Verma modules of singular infinitesimal character; there are several ways of generating these, however, the Penrose transform [5] being particularly good. For example, the series of conformally invariant powers of the Laplacian in Example 4.5.3 may be continued to allow $p \geq-n+1$, and there is a second order operator

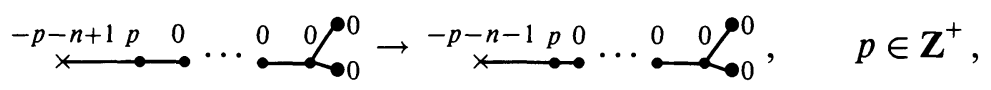

sending a totally symmetric $p$-form of conformal weight $p-n+1$ to one of weight $p-n-1$; a formula for the curved space extension of this is given in [37].

4.7. The translation principle. One of the ways of generating new homomorphisms from old is by means of the Jantzen-Zuckerman translation functor [36]. This depends on decomposing a $\mathscr{U}(\mathbf{g})$-module as a direct sum of submodules each of which is a generalized eigenvspace for the action of the center $\mathscr{Z}(\mathbf{g})$ of $\mathscr{U}(\mathbf{g})$ :

$$
V=V_{\lambda_{1}} \oplus V_{\lambda_{2}} \oplus \cdots \oplus V_{\lambda_{k}}, \quad \lambda_{i} \in \mathbf{h}_{\mathbf{R}}^{*}
$$

Thus $V_{\lambda}$ is a submodule on which $\left(z-\xi_{\lambda_{i}}(z)\right)^{d}$ acts by zero for all $z \in \mathscr{Z}(\mathbf{g})$, where $\xi_{\lambda_{i}}$ is the infinitesimal character of the Verma module $M_{\mathbf{b}}\left(\lambda_{i}\right)$. Any finitely generated g-module admits such a decomposition and the $\lambda_{i}$ are in distinct affine orbits of the Weyl group. (Recall that $\xi_{\lambda}=\xi_{w \cdot \lambda}$ for every $w \in W_{\mathbf{g}}$.) Let $P_{\lambda}$ denote the projection onto $V_{\lambda}$ and let $M_{\lambda}$ be the full subcategory of finitely generated g-modules on which $P_{\lambda}$ is the identity. This is usually called the category of g-modules of infinitesimal character $\xi_{\lambda}$.

Now we can define the translation functor. Let $\mu, \lambda$ be weights such that $\mu-\lambda$ is integral and let $F(\mu-\lambda)$ be the finite-dimensional g-module with extremal weight $\mu-\lambda$. Define

$$
\psi_{\mu}^{\lambda} V=P_{\mu}\left(F(\mu-\lambda) \otimes P_{\lambda} V\right)
$$

so that $\psi_{\mu}^{\lambda}: \mathrm{T}_{\lambda} \rightarrow \mathrm{T}_{\mu}$. The standard theorem on these functors [36] states that if both $\lambda$ and $\mu$ are dominant, then $\psi_{\mu}^{\lambda}$ and $\psi_{\lambda}^{\mu}$ are inverse functors, providing equivalences of categories. In other words, to understand homomorphisms of Verma modules in general regular integral character it 
suffices to understand these homomorphisms for trivial infinitesimal character, given by $\lambda=0$. For us, this means that invariant operators between forms on a conformal manifold generate all others of integral regular infinitesimal character, by translation.

We shall now carry this out in some detail. The reason for doing this is that we shall need some detailed control over the structure of these homomorphisms when we introduce conformal curvature.

First define an algebra $A(\mathbf{g})$ to be

$$
A(\mathbf{g})=\mathrm{T} \mathbf{g} /\langle\{x \otimes y-y \otimes x-[x, y] \mid x \in \mathbf{p}, y \in \mathbf{g}\}\rangle
$$

(where \langle\rangle means "ideal generated by"). This will be needed in the next section where the Cartan connection preserves only commutators of the form $[x, y]$ for $x \in \mathbf{p}$ and $y \in \mathbf{g} . \mathscr{U}(\mathbf{g})$ is a quotient of $A(\mathbf{g})$ which is in turn a $\mathscr{U}(\mathbf{p})$-bimodule. Evidently, as a vector space $A(\mathbf{g}) \cong \mathrm{T}\left(\mathbf{u}_{-}\right) \otimes \mathscr{U}(\mathbf{p})$, where $\mathbf{g}=\mathbf{p} \oplus \mathbf{u}_{-}$, and one may form induced modules which over Verma modules as left p-modules:

$$
A(\mathbf{g}) \otimes_{\mathscr{U}(\mathbf{p})} F_{\mathbf{p}}(\lambda) \rightarrow M_{\mathbf{p}}(\lambda) .
$$

Maximal elements are defined in $A(\mathbf{g}) \otimes_{\mathscr{U}(\mathbf{p})} F_{\mathbf{p}}(\lambda)$ and must cover maximal elements in $M_{\mathrm{p}}(\lambda)$. The important (and remarkable) fact is that the reverse is true, except for the extremal or "long" case.

Lemma 4.7.1. Let $\lambda$ be a dominant integral for

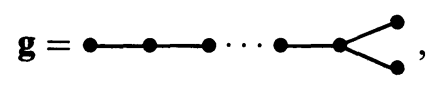

let

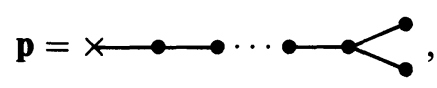

and let $w, w^{\prime} \in W^{\mathbf{p}}$. If

$$
D: M_{\mathbf{p}}\left(w^{\prime} \cdot \lambda\right) \rightarrow M_{\mathbf{p}}(\omega \cdot \lambda)
$$

is a homomorphism of Verma modules, then the image of $M_{\mathbf{p}}\left(w^{\prime} \cdot \lambda\right)$ is generated by a maximal vector $v$ which can be lifted to a maximal vector in $A(\mathbf{p}) \otimes_{\mathscr{U}(\mathbf{p})} F_{\mathbf{p}}(w \cdot \lambda)$, unless $l\left(w^{\prime}\right)=2 n, w=\mathrm{id}$.

A similar lemma holds in odd dimensions.

Proof. We need to write an expression for $v$ which is a sum of terms of the form $P \otimes v^{\prime}$ (where $P \in \mathrm{T}\left(\mathbf{u}_{-}\right)$and $v^{\prime} \in F_{\mathrm{p}}(w \cdot \lambda)$ ) and whose maximality can be proved using only commutators of the form $[x, y]$ for $x \in \mathbf{p}$ and $y \in \mathbf{g}$. Put $|\lambda|=\sum\left\langle\lambda, \alpha_{i}^{\vee}\right\rangle$ for $\left\{\alpha_{i}\right\}$ a set of simple roots for g. We proceed by induction on $|\lambda|$. 
Induction step. Let $\lambda_{i}$ be dual to $\alpha_{i}^{\vee}$ under the Killing form so that $\left\langle\lambda_{i}, \alpha_{j}^{\vee}\right\rangle=\delta_{i j}$. We may suppose that $\lambda=\mu+\lambda_{j}$ for some $j$ and $\mu$ dominant. Let $u \in M_{\mathrm{p}}(w \cdot \mu)$ be maximal of weight $w^{\prime} \cdot \mu$ and satisfy the condition of the lemma.

To find $v$ we use translation by the g-module $F\left(\lambda_{j}\right)$. As a p-module, this has a composition series as follows:

$$
\begin{aligned}
0 & \rightarrow F_{\mathbf{p}}\left(\lambda_{j}\right) \rightarrow F\left(\lambda_{j}\right) \rightarrow Q_{1} \rightarrow 0, \\
0 & \rightarrow \bigoplus_{i} F_{i}^{1} \rightarrow Q_{1} \rightarrow Q_{2} \rightarrow 0 \\
0 & \rightarrow \bigoplus_{i} F_{i}^{k} \rightarrow Q_{k} \rightarrow Q_{k+1} \rightarrow 0,
\end{aligned}
$$

etc., where each $F_{i}^{k}$ is an irreducible p-module of highest weight $\lambda_{i k}$. Furthermore, if $\mathbf{p}=\mathbf{l} \oplus \mathbf{u}$ is the Levi decomposition of $\mathbf{p}$ with $\mathbf{l}$ reductive and if $e \in \mathbf{h}^{*}$ is the unique element in the center of 1 such that ad $e$ acts by 1 on $\mathbf{u}$, then $\lambda_{j}(e)-\lambda_{i k}(e)=k$. Thus $k$ denotes the level of $F_{i}^{k}$ in the natural 1 grading of $F\left(\lambda_{j}\right)$. Now tensor through by $F_{\mathbf{p}}\left(w^{\prime} \cdot \mu\right)$ and apply $\mathscr{U}(\mathbf{g}) \otimes_{\mathscr{U}(\mathbf{p})}$ and finally apply $P_{\lambda}$ to project out the desired new infinitesimal character. To deduce $v$, let $\alpha$ be a highest weight vector for $F_{\mathbf{p}}\left(w^{\prime} \lambda\right)$, which occurs as one of the $F_{i}^{k}$ 's. Then $u \otimes \alpha \in \mathscr{U}(\mathbf{g}) \otimes_{\mathscr{U}(\mathbf{p})}\left(F_{\mathbf{p}}\left(w^{\prime} \cdot \mu\right) \otimes F_{i}^{k}\right)$. Push this forward to obtain

$$
u_{k} \in \mathscr{U}(\mathbf{g}) \otimes_{\mathscr{U}(\mathbf{p})}\left(F_{\mathbf{p}}\left(w^{\prime} \cdot \mu\right) \otimes Q_{k}\right)
$$

which is g-maximal modulo $\sum_{l<k} \sum_{i} F_{i}^{l}$. Because of infinitesimal character, we may lift $u_{k}$ to

$$
u_{k-1} \in \mathscr{U}(\mathbf{g}) \otimes_{\mathscr{U}(\mathbf{p})}\left(F_{\mathbf{p}}\left(w^{\prime} \cdot \mu\right) \otimes Q_{k-1}\right)
$$

by adding terms of the form $y \otimes\left(Q(Y) u \otimes \alpha^{\prime}\right)$ where

1. $y \in \mathbf{u}_{-}$,

2. $Q(Y) \in \mathscr{U}(\mathbf{l})$,

3. $\alpha^{\prime} \in F_{i}^{(k-1)}$ for some $i$,

and $u_{k-1}$ is g-maximal modulo $\sum_{l<k-1} \sum_{i} F_{i}^{l}$. Continuing this process ultimately yields the desired element

$$
v=u_{0} \in \mathscr{U}(\mathbf{g}) \otimes_{\mathscr{U}(\mathbf{p})}\left(F_{\mathbf{p}}\left(w^{\prime} \cdot \mu\right) \otimes F\left(\lambda_{j}\right)\right) .
$$

At each step $u_{n}$ is deduced from $u_{n+1}$ by a maximality requirement and this uniquely determines $u_{n}$. Notice that each lifting introduces only linear factors in $y \in \mathbf{u}_{-}$. 
We now need a further crucial

Lemma 4.7.2. $\quad F_{\mathbf{p}}\left(w^{\prime} \lambda_{j}\right) \subset \sum_{i} F_{i}^{1} \oplus \sum_{i} F_{i}^{2}$.

(This is easily proved by direct calculation, using the fact that $w^{\prime}=$ $\sigma_{1} \hat{w}$, where $\hat{w}$ lies in the Weyl group of $\mathbf{1}$.)

It follows that $u \otimes \alpha$ lifts to $v$ which can be expressed as a sum of terms of the form

$$
P(Q u) \otimes P^{\prime}\left(Q^{\prime} \theta\right)
$$

where $P, P^{\prime} \in \mathrm{T} \mathbf{u}_{-}$have degree at most two (in fact $\partial P+\partial P^{\prime} \leq 2$, $Q, Q^{\prime} \in \mathscr{U}(\mathbf{l})$, and $\theta$ is a highest weight vector in $\left.F\left(\lambda_{j}\right)\right)$. The maximality of such an expression depends only on the required commutators, given that $u$ is already maximal. That projection to the desired infinitesimal character does not depend on commutators in $u_{-}$follows from a splitting lemma analogous to 4.7.2.

Start of induction. It remains to prove that the induction can begin. This is evident (from the de Rham complex) for standard homomorphisms and so all that remains is to obtain the nonstandard operators between differential forms. It is easy to check that an invariant wave operator $\square: \mathscr{O}[-n+1] \rightarrow \mathscr{O}[-n-1]$ exists; the formula for the corresponding homomorphism of Verma modules is

$$
u \rightarrow 2\left(y_{1} y_{2 n}-y_{2} y_{2 n-1}+\cdots \pm y_{n} y_{n+1}\right) u_{0}
$$

for a suitable (null) basis of weight vectors $y_{i} \in \mathbf{u}_{-}$.

Put $F=F\left(\lambda_{1}\right)$ where

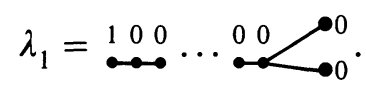

Repeated translation by $F$ yields operators

$$
\square^{p}: \mathscr{O}[-n+p] \rightarrow \mathscr{O}[-n-p] \text { for } 1 \leq p \leq n-1 .
$$

The next translation, for $p=n-1$, is from singular to nonsingular infinitesimal character: on tensoring $F_{\mathbf{p}}\left(-\lambda_{1}\right), F_{\mathbf{p}}\left((-2 n+1) \lambda_{1}\right)$ with $F$ we obtain the following composition series of $\mathbf{p}$-modules:

$$
\begin{gathered}
0 \rightarrow F_{\mathbf{p}}(0) \rightarrow F\left(-\lambda_{1}\right) \otimes F \rightarrow Q_{1} \rightarrow 0, \\
0 \rightarrow F_{\mathbf{p}}\left(\mu_{1}\right) \rightarrow Q_{1} \rightarrow F_{\mathbf{p}}\left(-2 \lambda_{1}\right) \rightarrow 0
\end{gathered}
$$

and

$$
\begin{aligned}
0 \rightarrow F_{\mathbf{p}}\left((-2 n+2) \lambda_{1}\right) & \rightarrow F\left((-2 n+1) \lambda_{1}\right) \otimes F \rightarrow Q_{1}^{\prime} \rightarrow 0, \\
0 \rightarrow F_{\mathbf{p}}\left(\mu_{2}\right) & \rightarrow Q_{1}^{\prime} \rightarrow F_{\mathbf{p}}\left(-2 n \lambda_{1}\right) \rightarrow 0 .
\end{aligned}
$$


Here,

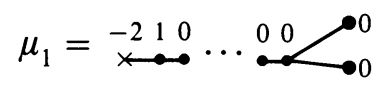

and

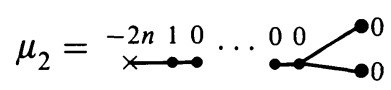

are the weights inducing the 1-forms and $(2 n-1)$-forms respectively. Applying $\mathscr{U}(\mathbf{g}) \otimes_{\mathscr{U}(\mathbf{p})}$ and projecting out infinitesimal characters we deduce the following diagram from the original homomorphism:

$$
\begin{aligned}
& \stackrel{0}{\rightarrow} M_{\mathbf{p}}(0) \rightarrow P_{0}\left(F \otimes M_{\mathbf{p}}\left(-\lambda_{1}\right)\right) \quad \rightarrow \quad M_{\mathbf{p}}\left(\mu_{1}\right) \rightarrow 0 \\
& 0 \rightarrow M_{\mathbf{p}}\left(\mu_{2}\right) \rightarrow P_{0}\left(F \otimes M_{\mathbf{p}}\left((-2 n+1) \lambda_{1}\right)\right) \rightarrow M_{\mathbf{p}}\left(-2 n \lambda_{1}\right) \rightarrow 0
\end{aligned}
$$

The composition $M_{\mathbf{p}}\left(\mu_{2}\right) \rightarrow M_{\mathbf{p}}\left(\mu_{1}\right)$ is nonzero and provides the nonstandard invariant differential $\Omega^{1} \rightarrow \Omega^{2 n-1}$. Further translation by $F$ leads to all the remaining operators $\Omega^{p} \rightarrow \Omega^{2 n-p}$ with $p \geq 2$. The operator $\mathscr{O} \mapsto \Omega^{2 n}$ does not arise by translation from simpler operators. We shall call this operator/homomorphism and those deduced from it by further translation, long (following a suggestion of Eastwood) since they correspond to the case $W^{1}=$ longest element of $W^{\mathbf{p}}$ and $w=$ id the shortest. Because these are excluded from 4.7.1 we will not be able to construct curved analogues of long operators.

\section{General conformal structures}

It is now time to extend the results of the previous sections to arbitrary conformal structures.

5.1. The central theorem. The construction of the previous section relies on the following differential-geometric ingredients. First, in order to be able to define homogeneous bundles a $P$-principal bundle structure $G \rightarrow G / P$ is required. Second, to identify the jet bundles as homogeneous bundles requires the identification of the Lie algebra $\mathbf{g}$ with the Lie algebra of left invariant vector fields on $G$. This is provided by the Maurer-Cartan form $\omega \in \Gamma\left(G, \mathbf{g} \otimes \Omega_{G}^{1}\right)$ which provides an isomorphism of each tangent space $\Theta_{g}$ with $\mathbf{g}$. Its inverse is an injective linear map

$$
\omega^{-1}: \mathbf{g} \rightarrow \Gamma(G, \Theta) \text {. }
$$

This is compatible with the $P$-principal structure when restricted to $\mathbf{p}$ and intertwines the adjoint representation of $\mathbf{p}$ on $\mathbf{g}$, namely,

$$
\omega^{-1}[v, w]=\left[w^{-1} v, w^{-1} w\right] \quad \forall v \in \mathbf{p}, w \in \mathbf{g} .
$$


It extends to a linear map of $\mathbf{p}$-modules

$$
\omega^{-1} \cdot \mathrm{Tg} \rightarrow \mathscr{D}_{G}
$$

from the tensor algebra on $\mathbf{g}$ to the differential operators on $G$. Because in fact $\omega^{-1}$ is a homomorphism of Lie algebras, this factors through an injection $\mathscr{U}(\mathbf{g}) \rightarrow \mathscr{D}_{G}$; in particular, the fibre of $\mathscr{D}_{G}$ at the identity is $\mathscr{U}(\mathbf{g})$, from which the results of the previous sections follow.

For a general conformal manifold $X$ there are no conformal motions; but an analogue of $G$ exists (at least in dimension $\geq 3$ ). This is a $P$ principal bundle $\mathscr{G} \rightarrow X$. The analogue of the Maurer-Cartan form is the normal admissible Cartan connection $\omega \in k \Gamma\left(\mathscr{G}, \Omega_{g}^{1} \otimes \mathbf{g}\right)$ which mimics the properties of the Maurer-Cartan form as follows:

1. $\omega_{g}: \Theta_{g} \mathscr{G} \rightarrow \mathbf{g}$ is an isomorphism for all $g \in \mathscr{G}$.

2. If $v \in \mathbf{p}$, then $\omega^{-1} v$ is the corresponding fiberwise vector field on $\mathscr{G}$.

3. For $p \in P$ and $R_{p}$ the right translation by $p$ on $\mathscr{G}, R_{p}^{*} \omega=$ $\operatorname{Ad}\left(p^{-1}\right) \omega$, or, infinitesimally, (16) holds.

$\mathscr{G}$ and $\omega$ are uniquely defined [4], [24], [29] (there is a small subtlety in three dimensions-see Remark 5.3.3). In particular, any $P$-module induces a bundle over $X$; thus $\mathscr{O}_{\mathbf{p}} \lambda$ is defined over $X$.

Again $\omega^{-1}$ extends as in equation (17):

$$
\omega^{-1}: \mathrm{Tg} \rightarrow \mathscr{D}_{g} .
$$

This time, however, $\omega^{-1}$ may not be a homomorphism of Lie algebras, so the mapping only factors through $A(\mathbf{g})$ by (3). Let $g \in \mathscr{G}$ be a fixed base point and examine the stalk $\mathscr{D}_{g}=\left(\mathscr{D}_{\mathscr{G}}\right)_{g}$. This is a quotient of $A(\mathbf{g})$ and we have morphisms of left $\mathbf{p}$-modules

$$
\begin{gathered}
A(\mathbf{g}) \otimes_{\mathscr{U}(\mathbf{p})} F_{\mathbf{p}}(\lambda) \rightarrow M_{\mathbf{p}}(\lambda) \\
\searrow \\
\mathscr{D}_{\mathscr{G}} \otimes_{\mathscr{U}(\mathbf{p})} F_{\mathbf{p}}(\lambda) .
\end{gathered}
$$

It now follows from Lemma 4.7.1 that any p-maximal element $\alpha$ in the Verma module can be lifted to an element of $A(\mathbf{g}) \otimes_{\mathscr{U}(\mathbf{p})} F_{\mathbf{p}}(\lambda)$ whose image in $\mathscr{D}_{g} \otimes_{\mathscr{U}(\mathbf{p})} F_{\mathbf{p}}(\lambda)$ is $\mathbf{p}$-maximal and so generates a p-submodule. Thus we have our main result:

Theorem 5.1.1. Let $X$ be a complex conformal manifold of dimension $m$ and let $\mathscr{G} \rightarrow X$ be the P-principal bundle determined by the conformal structure, where $P$ is a covering of the conformal Poincare group 
$\mathrm{CPO}(m, \mathbf{C})$. Let $F, F^{\prime}$ be P-modules. Then, apart from long homomorphisms, each homomorphism of Verma modules (of regular infinitesimal character) $M_{\mathbf{p}}\left(\lambda^{\prime}\right) \rightarrow M_{\mathbf{p}}(\lambda)$ yields an invariant differential operator

$$
\mathbf{D}: \mathscr{O}\left(F^{*}\right) \rightarrow \mathscr{O}\left(F^{\prime *}\right)
$$

depending only on the conformal structure of $X$.

Corollary 5.1.2. Every (regular) translation invariant differential operator on a flat conformal complex manifold of dimension at least three corresponds to a differential invariant of the conformal structure on an arbitrary conformal manifold of the same dimension, apart from long operators in even dimensions.

Remark 5.1.3. The symbols of the flat and corresponding curved differential operators formally have the same principal symbols; thus the operators have similar leading order terms, when expressed in terms of the Levi-Civita connection of a metric in the conformal class.

Remark 5.1.4. Eastwood and Rice [17] have established Corollary 5.1.2 in four dimensions using the local twistor bundle and the local twistor transport of R. Penrose [32]. Indeed, the local twistor bundle is the bundle induced from $\mathscr{G} \rightarrow X$ by a spinor representation of $\mathrm{SO}(6, \mathrm{C})$ restricted to $P . \mathscr{G}$ is a subbundle of its frame bundle and then local twistor transport is essentially the Cartan connection. Our argument is a formalization of their four-dimensional result. In that paper, and an earlier version of this one, it was mistakenly believed that all long operators also admit curved analogues. C. R. Graham has shown that no analogue of $\square^{3}: \mathscr{O}[1] \rightarrow$ $\mathscr{O}[-s]$ in four dimensions exists. Our error lies in supposing that the first long operator $\mathscr{O} \rightarrow \Omega^{2 n}$ could be obtained by translation from something simpler. Nonetheless, calculations of Graham, Mason and Sparling show that $\mathscr{O} \rightarrow \Omega^{2 n}$ does have a curved analogue.

It remains to construct these differential invariants explicitly in terms of the Levi-Civita connection and curvature of any metric in the conformal class. To do this it will be necessary to review briefly the construction of $\mathscr{G}$ and its Cartan connection $\omega$.

Remark 5.1.5. As we have said, the mapping

$$
\omega^{-1}: \mathbf{g} \rightarrow \Gamma\left(\mathscr{G}, \Theta_{g}\right)
$$

inverse to the Cartan connection $\omega$ of a conformal manifold is not in general a homomorphism of Lie algebras. The extent of its failure is the curvature $\Omega \in \Gamma\left(\mathscr{G}, \Omega_{\mathscr{G}} \otimes \mathbf{g}\right)$ defined by

$$
\Omega=d \omega+\frac{1}{2}[\omega, \omega] \text {. }
$$


so that for $u, v \in \mathbf{g}$

$$
\Omega\left(\omega^{-1} u, \omega^{-1} v\right)=[u, v]-\omega\left(\left[\omega^{-1} u, \omega^{-1} v\right]\right) .
$$

5.2. The construction of $\mathscr{G}$. Up to this point it has been useful to gloss over the connectivity of $G$ and $P$, assuming them to be simply connected so that the finite-dimensional representation theories of $P$ and $\mathbf{p}$ agree. In this section, however, $G=\mathrm{SO}(m+2, \mathbf{C})$ and $P=\mathrm{CPO}(m, \mathbf{C})=$ $L \ltimes \mathbf{C}^{m}$, where the conformal group $L=\mathrm{SO}(m, \mathbf{C}) \times \mathbf{C}^{\times}$corresponds to a reductive Levi factor $\mathbf{l} \subset \mathbf{p}$. Fix $\mathbf{g}=\mathbf{u}_{-} \oplus \mathbf{g}$.

For an $m$-dimensional manifold $X$, let $F^{i}(X)$ denote the $i$ th frame bundle of $X$ and let $G^{i}(m)=i$-frames at the origin of $\mathbf{C}^{m}$, i.e., the origin preserving diffeomorphisms of $\mathbf{C}^{m}$ modulo the normal subgroup consisting of those which are the identity to order $i$ at the origin. Then $G^{i}(m)$ is a Lie group and $F^{i}(X)$ is a $G^{i}(X)$-principal bundle over $X$. There is a natural epimorphism

$$
G^{i}(X) \rightarrow G^{1}(X) \cong \mathrm{GL}(m, \mathbf{C})
$$

which splits because the linear transformations are distinguished amongst the diffeomorphisms. The natural projection $F^{i+1}(X) \stackrel{\pi}{\longrightarrow} F^{i}(X)$ is compatible with $G^{i+1}(m) \stackrel{l}{\longrightarrow} G^{i}(m)$.

$F^{i}(X)$ comes equipped with a natural one-form $\Theta^{(i)}$-the canonical or soldering one-form-taking values in the Lie algebra $\mathbf{g}^{i-1}(m)$ of $G^{i-1}(m)$. For if $f \in F_{x}^{i}(X)$, then $f$ induces an isomorphism $f_{*}: \mathbf{g}^{i-1}(m) \rightarrow F_{\pi f}^{i-1}(X)$ and for $u \in \Theta_{f} F^{i}(X)$,

$$
\Theta^{(i)}(u)=\left(f_{*}\right)^{-1} \pi_{*} u .
$$

$\theta=\boldsymbol{\Theta}^{(1)}$ is the usual soldering form. Identifying $\mathbf{u}_{-} \cong \mathbf{C}^{m}$, obtain $\mathbf{g}^{1}(m) \cong \mathbf{u}_{-} \oplus \mathbf{g l}(m, \mathbf{C})$ and decompose $\Theta^{(2)}=\Theta_{-1} \oplus \Theta_{0}$. The properties of $\Theta^{i}$ are summarized in the following lemma [24].

Lemma 5.2.1. (i) If $v^{*}$ is a fiberwise vector field on $F^{i}(X)$ corresponding to $v \in \mathbf{g}^{i}(m)$, then $\Theta^{(i)}\left(v^{*}\right)=l_{*} v$.

(ii) If $g \in G^{i}(m)$ and $R_{g}$ denotes right translation on $F^{i}(X)$, then $R_{g}^{*} \Theta^{(i)}=\operatorname{Ad}\left(l(g)^{-1}\right) \Theta$.

(iii) $\pi^{*} \theta=\Theta_{-1}$ and there is a structure equation

$$
d \Theta_{-1}+\left[\Theta_{0}, \Theta_{-1}\right]=0
$$


Now a conformal structure on $X$ is equivalent to a distinguished $L$ principal subbundle $\mathscr{Q} \subset F^{1}(X) . \mathscr{G}$ is constructed, following [29], by finding a natural extension of $\mathscr{Q}$ to a $P$-principal subbundle of $F^{2}(X)$.

A local section s: $F^{1}(X) \rightarrow F^{2}(X)$ is admissible if, for $g \in \mathrm{GL}(m, \mathbf{C})$ and $f \in F^{1}(X)$,

$$
s(f \cdot g)=s(f) \cdot g
$$

(using the splitting of (18)). Kobayashi [24] has shown

Theorem 5.2.2. There is a one-to-one correspondence between local torsion free connections $\Gamma$ on $F^{1}(X)$ and local admissible sections $s_{\Gamma}$ by $\Gamma=s_{\Gamma}^{*} \Theta_{0}$.

Since the Levi-Civita connection of a metric in the conformal class is torsion free and preserves $\mathscr{Q}, \mathscr{Q}$ may be lifted locally to $\mathscr{Q}(\Gamma)=s_{\Gamma} \mathscr{Q}$ which, by virtue of the admissibility of $s_{\Gamma}$, is a $\mathrm{CO}(m, \mathbf{C})$-principal subbundle of $F^{2}(X)$. Now [3], [29]

Lemma 5.2.3. $P$ is a subgroup of $G^{2}(n)$.

Then, at least locally, the orbit $\mathscr{G}(\Gamma)$ of $\mathscr{Q}(\Gamma)$ under $P$ gives a $P$ principal bundle over $X$. To construct $G$ globally it is sufficient to show that $\mathscr{G}(\Gamma)$ is independent of the choice of $\Gamma$.

To do this recall the bigraded Spencer cohomology of $\mathbf{g}$ with respect to the |1|-grading

$$
\mathbf{g}_{-1}=\mathbf{u}_{-}, \quad \mathbf{g}_{0}=\mathbf{l}, \quad \text { and } \quad \mathbf{g}_{1}=\mathbf{u}
$$

so that $\mathbf{g}=\mathbf{g}_{-1} \oplus \mathbf{g}_{0} \oplus \mathbf{g}_{1}$ and $\left[\mathbf{g}_{i}, \mathbf{g}_{j}\right] \subset \mathbf{g}_{i+j}$. Define

$$
C^{p, q}\left(\mathbf{u}_{-}, \mathbf{g}\right)=\mathbf{g}_{p-1} \otimes \bigwedge^{q}\left(\mathbf{u}_{-}\right)^{*}
$$

and $\partial: C^{p, q} \rightarrow C^{p-1, q+1}$, satisfying $\partial^{2}=0$, by the formula

$$
\partial c\left(u_{0}, u_{1}, \cdots, u_{p}\right)=\sum_{j=1}^{p}(-1)^{j}\left[u_{j}, c\left(u_{0}, \cdots, \widehat{u}_{j}, \cdots, u_{p}\right)\right] .
$$

Spencer cohomology $H^{p, q}\left(\mathbf{u}_{-}, \mathbf{g}\right)$ is the (bigraded) cohomology of this complex. Observe that the adjoint action of the reductive group $L$ intertwines $\partial$ and so descends to an action on cohomology. The cohomology is easily computed using Kostant's theorem [36]; in particular we have

Lemma 5.2.4. If $\mathbf{g}=\mathbf{s o}(m+2, \mathbf{C})$ with $m \geq 4$ and if $\mathbf{g}$ is $|1|$-graded with respect to the conformal Poincaré parabolic subalgebra $\mathbf{p}$ as at (20), then $H^{1,1}\left(\mathbf{u}_{-}, \mathbf{g}\right)=H^{2,1}\left(\mathbf{u}_{-}, \mathbf{g}\right)=0$.

Let $\Gamma, \Gamma^{\prime}$ be torsion free connections on $F^{1}(X) . \mathscr{G}(\Gamma)=\mathscr{G}\left(\Gamma^{\prime}\right)$ if and only if there is a u-valued function $f$ on $\mathscr{Q}$ with $s_{\Gamma}=s_{\Gamma^{\prime}} \cdot \exp (f)$; in this case, $\mathscr{G}(\Gamma)$ and $\mathscr{G}\left(\Gamma^{\prime}\right)$ are called equivalent [29]. 
Theorem 5.2.5. Locally on $X$, equivalence classes of $\mathbf{g}(\Gamma)$ are parametrized by sections of the bundle induced over $X$ by the L-principal bundle $\mathscr{Q}$ and the representation of $L$ on $H^{1,1}\left(\mathbf{u}_{-}, \mathbf{g}\right)$.

Corollary 5.2.6. Over a complex conformal manifold $X$ of dimension at least four, there is a naturally defined P-principal subbundle $\mathscr{G} \subset F^{2}(X)$.

Remark 5.2.7. If $P, L$ are replaced by simply connected covering groups, then the obstruction to lifting $\mathscr{Q}$ and $\mathscr{G}$ to principal bundles with the new structure groups is the second Stiefel-Whitney class of $X$. Since differential operators are local, it is no loss of generality to suppose this vanishes and so to suppose, where necessary, that the structure group of $\mathscr{G}$ is again simply connected.

This is a good point to recall the Hodge theory of Spencer cohomology which will be required in the next section. Pick any basis $\left\{y_{i}\right\}$ of $\mathbf{u}_{-}$and let $\left\{x_{i}\right\}$ be the dual basis of $\mathbf{u}$ under the Killing form. Define $\partial^{*}: C^{p, q} \rightarrow$ $C^{p+1, q-1}$ by

$$
\partial^{*} c\left(u_{1}, \cdots, u_{q-1}\right)=\sum_{j=1}^{m}\left[x_{j}, c\left(y_{j}, u_{1}, \cdots, u_{q-1}\right)\right] .
$$

$\partial^{*}$ is of course independent of the choice of basis. It is possible to equip g with a Hermitian form so that $\partial^{*}$ is the adjoint of $\partial$ [25]. This leads to a Hodge theory for $H^{*}\left(\mathbf{u}_{-}, \mathbf{g}\right)$. Define $\square=\partial^{*} \partial+\partial \partial^{*}$; then there is a unique harmonic representative $f$, satisfying $\square f=0$, in each cohomology class of $H^{p, q}\left(\mathbf{u}_{-}, \mathbf{g}\right) . \square$ acts by scalars on irreducible representations of $\mathbf{1}$. Specifically, if $F=F_{\mathrm{p}}(\lambda)$ is the irreducible representation of 1 with highest weight $\mu$ and if $F^{*}$ occurs in $H^{*, *}\left(\mathbf{u}_{-}, \mathbf{g}\right)$ then $\square$ acts on $F^{*}$ by the scalar

$$
\frac{1}{2}(\langle\Lambda+\rho, \Lambda+\rho\rangle-\langle\mu+\rho, \mu+\rho\rangle),
$$

where $\rho$ is the semisum of the positive roots of $\mathbf{g}$ and $\Lambda$ is the highest root of $\mathbf{g}$.

5.3. The construction of $\omega$. With respect to the decomposition (20), any Cartan connection $\omega$ on $\mathscr{G}$ and its curvature $\Omega$ may be decomposed as

$$
\omega=\omega_{-1} \oplus \omega_{0} \oplus \omega_{1}, \quad \Omega=\Omega_{-1} \oplus \Omega_{0} \oplus \Omega_{1} .
$$

Notice that this decomposition is not p-invariant, but only l-invariant. For $p \in P$ and $R_{p}^{*}$ the right translation action of $P$ on $\mathscr{G}$,

$$
R_{p}^{*} \omega=\operatorname{Ad}\left(p^{-1}\right) \omega \text { and } R_{p}^{*} \Omega=\operatorname{Ad}\left(p^{-1}\right) \Omega
$$

by virtue of condition (3) in $\S 5.1$. This also implies that if $u^{*}$ is a fiberwise vector field on $\mathscr{G}$, so that $\Theta_{-1}\left(u^{*}\right)=0$, then $\Omega\left(u^{*}, \cdot\right)=0$. 
The admissible Cartan connections are those of the form

$$
\omega=\Theta_{-1} \oplus \Theta_{0} \oplus \omega_{1}
$$

(restricting $\Theta_{-1}$ and $\Theta_{0}$ to $\mathscr{G}$, the latter now taking values in l). $\omega_{1}$ is a u-valued one-form on $\mathscr{G}$. For such $\omega$, by virtue of the structure equation (19), $\Omega_{-1}=0$ and $\Omega_{0}$ satisfies

$$
R_{p}^{*} \Omega_{0}=\operatorname{Ad}\left(p^{-1}\right) \Omega_{0} \bmod \mathbf{u} .
$$

Let $u, v \in \mathbf{u}_{-1}$ and define a $C^{1,2}\left(\mathbf{u}_{-}, \mathbf{g}\right)$-valued function $W$ on $\mathscr{G}$ by

$$
W(u, v)=\Omega_{0}\left(\omega^{-1} u, \omega^{-1} v\right) .
$$

Then $W$ transforms by

$$
R_{p}^{*} W=\widetilde{\mu}\left(p^{-1}\right) W,
$$

where $\tilde{\mu}$ is the irreducible representation of 1 on $C^{1,2}\left(\mathbf{u}_{-}, \mathbf{g}\right)$, extended trivially to $\mathbf{p}$. So $W$ defines a section of the bundle on $X$ induced by $\tilde{\mu}$ which will be denoted by the same symbol. It should be thought of as a generalization of the Weyl conformal curvature of $X$-this is made precise below.

Now consider $\partial W$. Supposing that $\omega(w) \in \mathbf{u}_{-}$, obtain

$$
\partial W\left(\omega^{-1} u, \omega^{-1} v, \omega^{-1} w\right)=\left[\Theta_{-1}, \Omega_{0}\right](u, v, w) .
$$

But then,

$$
\left[\Theta_{-1}, \Omega_{0}\right]=\left[\Theta_{-1}, d \Theta_{0}+\frac{1}{2}\left[\Theta_{0}, \Theta_{0}\right]+\left[\Theta_{-1}, \omega_{1}\right]\right] .
$$

The only piece of this which might make a contribution to the right-hand side of (22) is $\left[\Theta_{-1}, d \Theta_{0}\right]$. Differentiating the structure equation (19) shows this to be $\left[d \Theta_{-1}, \Theta_{0}\right]$ so that it, too, makes no contribution. So $\partial W=0$ and $W$ defines a class in $H^{1,2}\left(\mathbf{u}_{-}, \mathbf{g}\right)$.

Now the scheme is to show that this class is independent of $\omega_{1}$; for then, $\omega$ may be fixed as an invariant of the conformal structure of $X$ by adjusting $\omega_{1}$ so that $W$ becomes the unique harmonic representative in its class. Such an $\omega$ is called a normal Cartan connection. This readjustment is the crucial ingredient in giving explicit formulas for the differential invariants of Theorem 5.1.1. It is achieved in the following theorem [34] which depends on the fact that $H^{2,1}\left(\mathbf{u}_{-}, \mathbf{g}\right)=0$.

Theorem 5.3.1. If $\omega^{\prime}$ is any admissible Cartan connection on $\mathscr{G}$, the class of $W\left(\omega^{\prime}\right)$ in $H^{1,2}\left(\mathbf{u}_{-}, \mathbf{g}\right)$ is independent of $\omega^{\prime}$ and there exists a unique admissible Cartan connection $\omega$ with $W(\omega)$ harmonic. 
Proof. This is best taken in two steps:

Uniqueness of class. Let $\omega^{\prime}$ and $\omega^{\prime \prime}$ be two admissible Cartan connections on $\mathscr{G} ; \omega^{\prime-1}$ and $\omega^{\prime \prime-1}$ differ only on $\mathbf{u}$ and then by an element of $\omega^{\prime-1} \mathbf{u}$. Thus there exists a $C^{2,1}\left(\mathbf{u}_{-}, \mathbf{g}\right)$-valued function $f$ on $\mathscr{G}$ defined by

$$
u^{\prime}=u^{\prime \prime}=\omega^{\prime-1} f(u)
$$

where $\omega^{\prime}\left(u^{\prime}\right)=\omega^{\prime \prime}\left(u^{\prime \prime}\right)=u \in \mathbf{u}_{-}$. If $v, v^{\prime}, v^{\prime \prime}$ are similarly defined then the fact that $\Omega_{0}^{\prime}$ and $\Omega_{0}^{\prime \prime}$ are zero on $\omega^{\prime-1} \mathbf{p}$ implies that

$$
\begin{aligned}
\left\{W\left(\omega^{\prime}\right)-W\left(\omega^{\prime \prime}\right)\right\}(u, v) & =\Omega_{0}^{\prime}\left(u^{\prime}, v^{\prime}\right)-\Omega_{0}^{\prime \prime}\left(u^{\prime \prime}, v^{\prime \prime}\right)=\left(\Omega_{0}^{\prime}-\Omega_{0}^{\prime \prime}\right)\left(u^{\prime \prime}, v^{\prime \prime}\right) \\
& =\left[\Theta_{-1}, \omega_{1}^{\prime}-\omega_{1}^{\prime \prime}\right]\left(u^{\prime \prime}, v^{\prime \prime}\right)=\partial f(u, v) .
\end{aligned}
$$

Existence and uniqueness of $\omega$. To establish the existence of an admissible Cartan connection, locally pick a connection $\Gamma$ so that $\mathscr{G}=\mathscr{G}(\Gamma)$. Let $g=q a \in \mathscr{G}(\Gamma)$, where $q \in s_{\Gamma} \mathscr{Q}$ and $a \in \exp \mathbf{u}$. If $Y \in \Omega_{g}(\mathscr{G})$, then uniquely

$$
Y=\left(R_{a}\right)_{*} X+x^{*}
$$

for $X \in \Theta_{q}\left(s_{\Gamma} \mathscr{Q}\right)$ and $x^{*}$, the fiberwise vector field corresponding to some $x \in \mathbf{u}$. Define an admissible $\omega^{\prime}$ by setting

$$
\omega_{1}^{\prime}(X)=0, \quad \omega^{\prime}(Y)=\operatorname{Ad}\left(a^{-1}\right)\left(\omega^{\prime}(X)\right)+x .
$$

It is straightforward to check that $\omega^{\prime}$ so defined is a Cartan connection. It should be thought of as a lifting of the connection $\Gamma$ which in the sequel will be the Levi-Civita connection of some metric in the conformal class. To construct a normal Cartan connection let

$$
\square f=-\partial^{*} W\left(\omega^{\prime}\right) \text {. }
$$

Since, by Lemma $5.2 .4, H^{2,1}\left(\mathbf{u}_{-}, \mathbf{g}\right)=0$, this has a unique solution for $f$. So define admissible $\omega$ by

$$
\omega^{-1} y=\omega^{\prime-1} y+\omega^{\prime-1} f(y)
$$

for $y \in \mathbf{u}_{-}$. Then, as in the first part of the proof,

$$
W(\omega)=\partial f+W\left(\omega^{\prime}\right)
$$

so that $\partial W(\omega)=\partial^{*} W(\omega)=0$ and $\square \omega=0$. It is easy to check that $\omega$ is a Cartan connection.

Finally, since the solution of (23) is unique, $\omega$ is unique and globally defined. q.e.d. 
Remark 5.3.2. The class of $W(\omega)$ defines a section of the bundle on $X$ induced by $H^{1,2}\left(\mathbf{u}_{-}, \mathbf{g}\right)$; this is the Weyl conformal curvature of $X$. In even and odd dimensions above five this is a section of the bundles

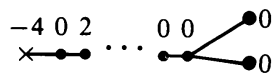

and

$$
\underset{+402}{-40} \stackrel{0}{\longrightarrow} .
$$

If four dimensions, the Weyl curvature has two irreducible components:

$$
\stackrel{4-40}{\longrightarrow \sim} \oplus \stackrel{0-4}{\longrightarrow} \stackrel{0}{\longrightarrow \sim} \longrightarrow
$$

whilst in five dimensions, the Weyl curvature is a section of

$$
\begin{array}{lll}
-40 & 4 \\
\end{array}
$$

Remark 5.3.3. The case of three dimensions is special; for one $H^{2,1}\left(\mathbf{u}_{-}, \mathbf{g}\right)$ does not vanish but $H^{1,2}\left(\mathbf{u}_{-}, \mathbf{g}\right)$ does! It turns out that a unique Cartan connection does exist [4], although the methods of [29] are not sufficient to establish this. Only $H^{2,2}\left(\mathbf{u}_{-}, \mathbf{g}\right)$ is nonzero, amongst second cohomology. This is related to the fact that in three dimensions the Weyl tensor is replaced by a third order invariant tensor, the Cotton-York tensor.

5.4. Explicit computations. Let $\Gamma$ be the Levi-Civita connection of some metric in the conformal class on $X$, and let $\omega^{\prime}$ be the admissible Cartan connection on $\mathscr{G}$ constructed from it in Theorem 5.3.1. Let $\omega$ be the unique normal Cartan connection on $\mathscr{G}$ and for $y \in \mathbf{u}_{-}$, define vector fields $y^{\prime}, y^{*}$ on $\mathbf{g}$ by $\omega^{\prime}\left(y^{\prime}\right)=\omega\left(y^{*}\right)=y$. With $f$ defined as in the second part of the proof of Theorem 5.3.1, $f(y) \in \mathbf{u}$ and

$$
y^{*}=y^{\prime}+\omega^{-1}(f(y)) \text {. }
$$

If $\varphi: M_{\mathrm{p}}(\mu) \rightarrow M_{\mathrm{p}}(\lambda)$ is a homomorphism of Verma modules, then $\varphi$ is determined by a mapping of a highest weight vector $\gamma$ of $M_{\mathbf{p}}(\mu)$ to a maximal vector in $M_{\mathbf{p}}(\lambda)$ of weight $\mu$ of the form $\sum p^{i}\left(y_{j}\right) \kappa_{i}$, where $\left\{\kappa_{i}\right\}$ is a weight basis of $F_{\mathbf{p}}(\lambda)$ and the $p^{i}$ are homogeneous polynomials in the $y_{j}$ which form a roots space basis of $\mathbf{u}_{-}$. These must be chosen according to Lemma 4.7.1. To obtain a differential operator, each $y_{i}$ must be replaced by a vector field $y_{j}^{*}$.

To obtain differential operators expressed in terms of the Levi-Civita connection $\Gamma$, simply substitute the $y_{i}^{*}$ in terms of $y_{i}^{\prime}$ using (26), noting that the $y_{i}^{\prime}$ will induce the differential operator obtained from projecting $\nabla_{a_{1}} \cdots \nabla_{a_{n}} \psi$ into its irreducible factor $\mathscr{O}_{\mathbf{p}}(\mu)$, where $\psi$ is a section of 
$\mathscr{O}_{p}(\lambda)$ and $\nabla$ is the covariant differential of $\Gamma$. The terms $\omega^{-1}\left(f\left(y_{i}\right)\right)$ will construct suitable correction terms needed to make the resulting differential operator invariant.

To compute $f$, let $s_{\Gamma}$ be the (local) admissible section associated to $\Gamma$ by Kobayashi's Theorem 5.2.2. Let $R(\Gamma)$ denote the curvature form of $\Gamma$ on $\mathscr{Q}$. Then, on $\mathscr{Q}$,

$$
s_{\Gamma} \Omega_{0}=R(\Gamma)+\left[\theta, s_{\Gamma}^{*} \omega_{1}\right]
$$

Thus if $\widetilde{u}$ and $\widetilde{v}$ are horizontal vector fields on $\mathscr{Q}$ with $\theta(\widetilde{u})=u$ and $\theta(\widetilde{v})=v$, then

$$
W(u, v)=s_{\Gamma}^{*} \Omega(\widetilde{u}, \widetilde{v}) .
$$

By means of the fact that $s_{\Gamma}^{*} \omega_{1}^{\prime}=0$ and $\Gamma=s_{\Gamma}^{*} \Theta_{0}$, this yields

$$
W(u, v)=R(\Gamma)(\widetilde{u}, \widetilde{v})+\partial f(u, v) .
$$

Writing $r(\Gamma)(u, v)=R(\Gamma)(\widetilde{u}, \widetilde{v})$ and making use of the normality of $\omega$ together with $H^{2,1}\left(\mathbf{u}_{-}, \mathbf{g}\right)=0$, we have

$$
f=-\square^{-1} \partial^{*} r(\Gamma)
$$

Thought of as a section of an induced bundle over $X, \partial^{*} r(\Gamma)$ is the Ricci curvature of $\Gamma[29]$ and so $f$ is easily obtained, using the scalars of (21).

Remark 5.4.1. (i) The correction terms computed from $f$ above involve only the Ricci curvature of $\Gamma$ and its derivatives. So the differential invariants may be expressed in a form involving only the Levi-Civita connection of a metric in the conformal class and its Ricci curvature. The formal expressions for the invariants will always appear as the same combination of covariant derivatives and Ricci curvature, irrespective of $\Gamma$.

(ii) The highest order part of these operators is formally as in the flat case, the Levi-Civita connection replacing the flat connection. If $\left\{Y_{j}\right\}$ is a basis of the negative root spaces of $\mathbf{1}$, then in an expansion of an expression of the form

$$
y_{i_{1}}^{*} y_{i_{2}}^{*} \cdots y_{i_{n}}^{*}\left(Y_{j_{1}} Y_{j_{2}} \cdots Y_{j_{p}} \gamma\right)
$$

in terms of $y_{j}^{\prime}$,

(a) $y_{i_{n}}^{*}$ gives rise to no curvature correction terms,

(b) for each occurence of a curvature correction term in the expansion there are two fewer occurrences of $y_{i}^{\prime}$ than $y_{i}^{*}$ in the original expression.

It follows from (a) that all first order invariant differential operators can include no curvature terms and are formally as in the homogeneous case, as originally proved in [21]. From (b) it is clear that the first curvature 
correction term is associated to a differential operator of order at least two less than that of the leading term.

It is easiest to display further properties of the construction in the context of an actual example.

\section{Calculations in four dimensions}

Let $X$ be a four-dimensional complex conformal manifold. Locally, pick a metric in the conformal class and let $\Gamma$ be its Levi-Civita connection. The aim of this section is to compute the differential invariants of the conformal structure of $X$ in terms of the covariant derivative $\nabla$ of $\Gamma$. As in $\S 3$, free use will be made of the abstract index notation of $\mathbf{R}$. Penrose [31].

Now $r(\Gamma)$ is an $\left(\mathbf{l} \otimes \Lambda^{2}\left(\mathbf{u}_{-}\right)^{*}\right)$-valued function on the $\mathrm{CO}(4, \mathbf{C})$-principal bundle $\mathscr{Q}$; it represents a section of the induced bundle

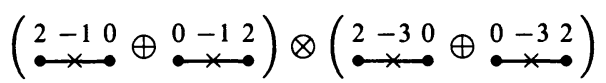

on $X$. Because the Levi-Civita connection is torsion free, the projections of $r(\Gamma)$ into certain of the irreducible components of this bundle vanish; $r(\Gamma)$ is actually a section of

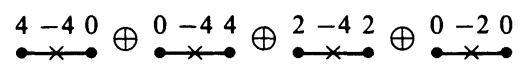

(comprising self- and anti-dual Weyl curvature, tracefree Ricci curvature and scalar curvature). (The Spencer cohomology $H^{1,2}\left(\mathbf{u}_{-}, \mathbf{g}\right)$ induces the bundle $\stackrel{4-40}{\rightarrow} \oplus \stackrel{0-44}{\longrightarrow}$. $)$ Then $\partial^{*} r(\Gamma)$ gives a section of

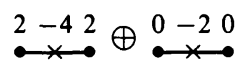

i.e., Ricci curvature, as claimed, since $\partial^{*}$ intertwines the action of 1 . Write this as

$$
\left.R_{a b=\left(R_{a b}\right.}-\frac{1}{4} R g_{a b}\right) \oplus \frac{1}{4} R g_{a b}=-2 \Phi_{a b} \oplus 6 \Lambda g_{a b} .
$$

$\square^{-1}$ acts by $\frac{1}{2}$ on $\stackrel{2-42}{\longrightarrow}$ and by $\frac{1}{6}$ on $\stackrel{0-20}{\longrightarrow}$ so that $f=-\square^{-1} \partial^{*} r(\Gamma)$ induces the tensor

$$
\mathscr{P}_{a b}=\Phi_{a b}-\Lambda g_{a b} \text {. }
$$

Recall that if $d \tilde{\mu}$ is the irreducible representation of $\mathbf{p}$ on $C^{1,2}\left(\mathbf{u}_{-}, \mathbf{g}\right)$, then

$$
u^{*} \cdot f=-d \widetilde{\mu}(u) f
$$

for $\omega\left(u^{*}\right)=u \in \mathbf{p}$. It follows that $f$ may be thought of as an element of the dual representation $\left[C^{1,2}\left(\mathbf{u}_{-}, \mathbf{g}\right)\right]^{*}$ under the differential action of 
$\mathbf{p}$, via $\omega_{-1}$. Split $f$ into its irreducible parts, and let $\Phi$ and $-\Lambda$ denote the highest weight components under the differential action of $\mathbf{p}$. Using the notation of $\S 3$, write

$$
f\left(y_{i}\right)=\sum \mathscr{P}_{i j} x_{j} \quad \text { so } y_{i}^{*}=y_{i}^{\prime}+\sum \mathscr{P}_{i j} x_{j}^{*} ;
$$

then $\mathscr{P}_{i j}$ is as in Table 1. (To form $\mathscr{P}_{i j}$, think of $\sum \mathscr{P}_{i j} x_{j}$ as an element

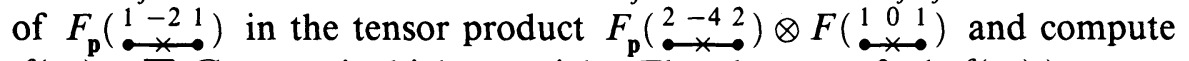
$f\left(y_{1}\right)=\sum \mathscr{P}_{1 j} x_{j}$ as its highest weight. Then lower to find $f\left(y_{j}\right)$.)

TABLE 1. Curvature correction terms $\mathscr{P}_{i j}$

\begin{tabular}{|c|r|r|r|r|}
\hline$j$ & 1 & 2 & 3 & 4 \\
\hline 1 & $\Phi$ & $-\frac{1}{2} Y_{1} \Phi$ & $\frac{1}{2} Y_{2} \Phi$ & $-\Lambda-\frac{1}{4} Y_{1} Y_{2} \Phi$ \\
\hline 2 & $-\frac{1}{2} Y_{1} \Phi$ & $\frac{1}{2} Y_{1}^{2} \Phi$ & $\Lambda-\frac{1}{4} Y_{1} y_{2} \Phi$ & $\frac{1}{4} Y_{1}^{2} Y_{2} \Phi$ \\
\hline 3 & $\frac{1}{2} Y_{2} \Phi$ & $\Lambda-\frac{1}{4} Y_{1} Y_{2} \Phi$ & $\frac{1}{2} Y_{2}^{2} \Phi$ & $-\frac{1}{4} Y_{1} Y_{2}^{2} \Phi$ \\
\hline 4 & $-\Lambda-\frac{1}{4} Y_{1} Y_{2} \Phi$ & $\frac{1}{4} Y_{1}^{2} Y_{2} \Phi$ & $-\frac{1}{4} Y_{1} Y_{2}^{2} \Phi$ & $\frac{1}{4} Y_{1}^{2} Y_{2}^{2} \Phi$ \\
\hline
\end{tabular}

It is now time for concrete examples.

6.1. Local twistor transport. This first example relates the present construction to local twistor transport [32]. Let $\mathscr{T}^{\alpha}$, the local twistor bundle, be induced by restricting the self-representation $T^{\alpha}$ of $\mathbf{g}=\mathrm{sl}(4, \mathrm{C})$ to p. Let $T_{\alpha}$ be the dual of this representation, using Penrose's abstract index notation. Then there is a homomorphism of induced modules

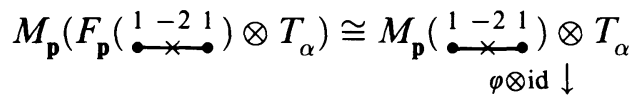

$$
\begin{aligned}
& M_{\mathbf{p}}(\mathbf{C}) \otimes T_{\alpha} \cong M_{\mathbf{p}}\left(\mathbf{C} \otimes T_{\alpha}\right)
\end{aligned}
$$

where $\mathbf{C}$ is the trivial module and $\varphi$ is generated by $y_{1} \rightarrow y_{1} \underline{1} ; \underline{1}$ and $y_{1}$ are highest weight vectors in $\mathbf{C}$ and $F_{\mathbf{p}}\left(\begin{array}{ll}1-2 & -1 \\ - & -\end{array}\right)$ respectively.

Let $T_{\alpha}$ be spanned by weight vectors as in Table 2. Fixing a local metric in the conformal class is equivalent to restricting to the action of $I^{s}=[1,1]$ on $T_{\alpha}$. Do this and so split the sequence

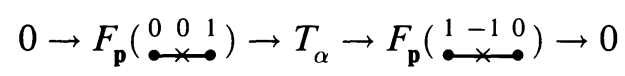

by thinking of the spans of

$$
\{\alpha, \beta\} \rightarrow\{\alpha, \beta, \gamma, \delta\} \rightarrow\{\gamma, \delta\} .
$$

Splitting $T_{\alpha} \otimes F_{\mathrm{p}}\left(\begin{array}{c}1-2 \\ -\sim\end{array}\right)$ obtains irreducibles $F_{\mathrm{p}}(\stackrel{1-2}{-2}) \oplus F_{\mathrm{p}}\left(\begin{array}{c}1-1 \\ -\sim\end{array}\right)$ and

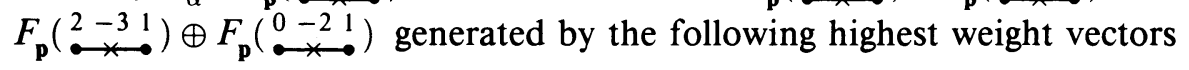


over $\mathscr{U}\left(\mathbf{l}^{s}\right)$ :

$$
\begin{aligned}
& y_{1} \otimes \alpha ; \frac{1}{2}\left(y_{1} \otimes \beta-y_{3} \otimes \alpha\right), \\
& y_{1} \otimes \gamma ; \frac{1}{2}\left(y_{1} \otimes \delta-y_{2} \otimes \gamma\right) .
\end{aligned}
$$

Under (28), for instance,

$$
y_{1} \otimes \alpha \stackrel{\varphi \otimes \mathrm{id}}{\longrightarrow}\left(y_{1} \underline{1}\right) \otimes \alpha \cong y_{1}(\underline{1} \otimes \alpha)-\underline{1} \otimes y_{1} \cdot \alpha=y_{1}(\underline{1} \otimes \alpha) .
$$

TABLE 2. Weight vectors in $T_{\alpha}$

\begin{tabular}{|l|l|l|l|}
\hline Vector & Weight & Vector & Weight \\
\hline$\alpha$ & $\stackrel{0}{0}-1$ & $\beta=Y_{2} \alpha$ & $011-1$ \\
\hline$\gamma=y_{1} \beta$ & $\stackrel{1}{1}-10$ & $\delta=Y_{1} \gamma$ & -100 \\
\hline
\end{tabular}

Replacing $y_{i}$ by $y_{i}^{*}$ written in terms of $y_{i}^{\prime}$, using Table $1,(28)$ gives the mapping induced by the direct sum of

$$
\begin{array}{ll}
y_{1} & \rightarrow y_{1}^{\prime} \alpha, \\
y_{1} \otimes \beta-y_{3} \otimes \alpha & \rightarrow y_{1}^{\prime} \beta-y_{3}^{\prime} \alpha-2 \gamma, \\
y_{1} \otimes \gamma & \rightarrow y_{1}^{\prime} \gamma-\mathscr{P}_{11} \beta+\mathscr{P}_{13} \alpha, \\
y_{1} \otimes \delta-y_{2} \otimes \gamma & \rightarrow y_{1}^{\prime} \delta-y_{2}^{\prime} \gamma-2\left(\mathscr{P}_{14} \alpha-\mathscr{P}_{12} \beta\right) .
\end{array}
$$

Overall, on induced bundles, this gives the conformally invariant operator

$$
\left(\omega^{A}, \pi_{\left.A^{\prime}\right)} \rightarrow\left(\nabla_{B B^{\prime}} \omega^{A}+\varepsilon_{B}^{A} \pi_{B^{\prime}}, \nabla_{B B^{\prime}} \pi_{A^{\prime}}-\mathscr{P}_{B B^{\prime} A A^{\prime}} \omega^{A}\right),\right.
$$

that is, local twistor transport.

6.2. The conformally invariant Laplacian. Recall from $\S 3$ that

$$
\left(y_{1} y_{4}+y_{4} y_{1}-y_{2} y_{3}-y_{3} y_{2}\right) \alpha
$$

induces the Laplacian $\square: \mathscr{O}[-1] \rightarrow \mathscr{O}[-3]$. Writing this in terms of $y_{i}^{*}$ and expanding obtain

$$
\left(y_{1}^{\prime} y_{4}^{\prime}+y_{4}^{\prime} y_{1}^{\prime}-y_{2}^{\prime} y_{3}^{\prime}-y_{3}^{\prime} y_{2}^{\prime}\right) \alpha-\left(\mathscr{P}_{14}+\mathscr{P}_{41}-\mathscr{P}_{23}-\mathscr{P}_{32}\right) \alpha
$$

which, on substituting for $\mathscr{P}_{i j}$ from Table 1 , yields the conformally invariant Laplacian

$$
\nabla^{a} \nabla_{a}+R / 6: \mathscr{O}[-1] \rightarrow \mathscr{O}[-3]
$$

6.3. The Bach tensor. The irreducible components of the Weyl conformal curvature are sections of the induced bundles 
usually called the self- and anti-self-dual Weyl spinors [32] and denoted by totally symmetric spinors $\widetilde{\psi}_{A^{\prime} B^{\prime} C^{\prime} D^{\prime}}$ and $\psi_{A B C D}$. By the general theory, there is a second order differential invariant $\stackrel{4-40}{\longrightarrow \rightarrow} \stackrel{2-62}{\rightarrow-}$ induced by the following expression:

$$
\begin{aligned}
\left(3 y_{2}+y_{1} Y_{1}\right)\left(4 y_{2}+y_{1} Y_{1}\right) \psi & \\
= & {\left[12 y_{2}^{2}+3 y_{1} y_{2} Y_{1}+3 y_{2} y_{1} Y_{1}+y_{1}^{2} Y^{2} 1_{1}\right] \psi, }
\end{aligned}
$$

where $\psi$ is an appropriate highest weight vector. Replacing $y_{i}$ by $y_{i}^{*}=$ $y_{i}^{\prime}+\sum \mathscr{P}_{i j} x_{j}$ in this expression yields

$$
\left(3 y_{2}^{\prime}+y_{1}^{\prime} Y_{1}\right)\left(4 y_{2}^{\prime}+y_{1}^{\prime} Y_{1}\right) \psi+\left(12 \mathscr{P}_{22}+6 \mathscr{P}_{12} Y_{1}+\mathscr{P}_{11} Y_{1}^{2}\right) \psi
$$

The terms involving $\mathscr{P}_{i j}$ simplify to

$$
\Phi\left(Y_{1}^{2} \psi\right)-3\left(Y_{1} \Phi\right)\left(Y_{1} \psi\right)+6\left(Y_{1}^{2} \Phi\right) \psi
$$

which induces the projection $\stackrel{2-42}{-4-40} \rightarrow \stackrel{2-62}{\longrightarrow}$ and so it follows that the invariant operator is given by the formula

$$
\varphi_{A^{\prime} B^{\prime} C^{\prime} D^{\prime}} \rightarrow\left(\nabla_{(A}^{C^{\prime}} \nabla_{B)}^{D^{\prime}}+\Phi_{A B}^{C^{\prime} D^{\prime}}\right) \varphi_{A^{\prime} B^{\prime} C^{\prime} D^{\prime}}
$$

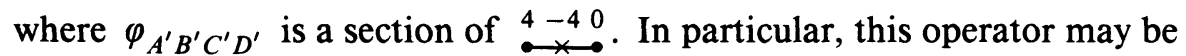
applied to the self-dual Weyl curvature; the resulting symmetric, tracefree tensor,

$$
B_{A B A^{\prime} B^{\prime}}=2\left(\nabla_{(A}^{C^{\prime}} \nabla_{B)}^{D^{\prime}}+\Phi_{A B}^{C^{\prime} C^{\prime}}\right) \widetilde{\psi}_{A^{\prime} B^{\prime} C^{\prime} D^{\prime}},
$$

is an invariant of the conformal structure of $X$ known as the Bach tensor [2], [27]. The anti-self-dual Weyl curvature is similarly the subject of an invariant differential operator; by symmetry this is obtained from the above operator by interchanging primed and unprimed indices. By virtue of the Bianchi identities, the invariant tensor so obtained is again the Bach tensor.

The Bach tensor is itself the subject of the first order invariant differential operator $\stackrel{2-62}{\rightarrow} \rightarrow \stackrel{1-61}{\longrightarrow}$ by $B_{a b} \rightarrow \nabla^{a} B_{a b}$. But, as a consequence of the Bianchi identity, this is zero and no new conformally invariant tensor is obtained.

6.4. Speeding calculation. The process just illustrated is certain to produce differential invariants of the conformal structure of $X$ from homomorphisms of Verma modules; of course, as the order of the operator increases, the calculation becomes tedious. Remark 5.4.1 suggests a more efficient method. Any "curvature correction term" in a differential invariant $\mathbf{D}: \mathscr{O}(F) \rightarrow \mathscr{O}\left(F^{\prime}\right)$ must be a combination of derivatives of $\Phi_{a b}$ and 
$\Lambda$ and must arise from a projection onto an irreducible subbundle in the tensor product:

$$
\left(\otimes^{i} \mathscr{O}_{\left(A^{\prime} B^{\prime}\right)(A B)}\right) \otimes\left(\otimes^{j} \mathscr{O}[-2]\right) \otimes\left(\otimes^{p} \Omega_{X}^{1}\right) \otimes \mathscr{O}(F) \rightarrow \mathscr{O}\left(F^{\prime}\right),
$$

where $2 i+2 j+p=$ order of $\mathbf{D}$. (Of course, several correction terms involving varying degrees of derivatives of $\Phi_{a b}$ and $\Lambda$ may arise from one such projection.) It is a simple matter to calculate highest weight vectors in such a tensor product; a suitable combination of these will give the terms arising from the substitution $y_{i}^{*}=y_{i}^{\prime}+\sum \mathscr{P}_{i j} x_{j}$. Clearly, this combination must be maximal under the action of $\mathbf{p}$; this is true if it is annihilated by $x_{1}$ (since it is already annihilated by $X_{1}$ and $X_{2}$ ). This condition determines the linear combination. All that remains is to obtain the correct multiple of this combination to add to the leading order term. This is most easily accomplished by a judicious partial expansion of the inducing maximal vector or by a judicious inspection of the terms generated by the leading order covariant differential under a conformal rescaling ([32] contains useful formulas for this purpose). The following two examples illustrate this technique.

6.5. A third order operator on spinors. The expression

$$
\varphi=\left(-y_{3}+y_{1} Y_{2}\right)\left(-2 y_{3}+y_{1} Y_{2}\right)\left(-3 y_{3}+y_{1} Y_{2}\right) \lambda
$$

induces a differential invariant $\stackrel{1-23}{\longrightarrow} \rightarrow \stackrel{4-50}{\rightarrow-4}$ whose leading order term is

$$
\lambda_{D^{\prime}(A B C)} \rightarrow \nabla_{\left(A^{\prime}\right.}^{A} \nabla_{C^{\prime}}^{B} \lambda_{\left.D^{\prime}\right) A B C}
$$

The only possibility in equation (31) with $i+j>0$ is $i=1, p=1$; accordingly, correction terms must have the form

$$
\left(\nabla_{\left(A^{\prime}\right.}^{A} \Phi_{B^{\prime} C^{\prime}}^{B C}\right) \lambda_{\left.D^{\prime}\right) A B C} \text { or } \Phi_{\left(A^{\prime} B^{\prime}\right.}^{A B} \nabla_{C^{\prime}}^{C} \lambda_{\left.D^{\prime}\right) A B C}
$$

corresponding to highest weight vectors

$$
\begin{aligned}
\chi= & \left(y_{1}^{\prime} \mathscr{P}_{11} l\right) Y_{2}^{3} \lambda-2\left(y_{1}^{\prime} \mathscr{P}_{13}\right) Y_{2}^{2} \lambda-\left(y_{3}^{\prime} \mathscr{P}_{11}\right) Y_{2}^{2} \lambda \\
& +\left(y_{3}^{\prime} \mathscr{P}_{13}\right) Y_{2} \lambda+2\left(y_{1}^{\prime} \mathscr{P}_{33}\right) Y_{2} \lambda-6\left(y_{3}^{\prime} \mathscr{P}_{33}\right) \lambda, \\
\tau= & \mathscr{P}_{11} y_{1}^{\prime} Y_{2}^{3} \lambda-2 \mathscr{P}_{13} y_{1}^{\prime} Y_{2}^{2} \lambda-\mathscr{P}_{11} y_{3}^{\prime} Y_{2}^{2} \lambda \\
& +4 \mathscr{P}_{13} y_{3}^{\prime} Y_{2} \lambda+2 \mathscr{P}_{33} y_{1}^{\prime} Y_{2} \lambda-6 \mathscr{P}_{33} y_{3}^{\prime} \lambda
\end{aligned}
$$

in the expansion of (32); requiring that $\chi+a \cdot \tau$ be annihilated by $x_{1}$ easily yields $a=2$ and so, if $\varphi^{*}$ and $\varphi^{\prime}$ denote $\varphi$ with $y_{i}$ replaced by $y_{i}^{*}$ or $y_{i}^{\prime}$, then

$$
\varphi^{*}=\varphi^{\prime}+b(\chi+2 \tau)
$$


To determine $b$, multiply $\varphi^{*}$ out; $\left(y_{3} \mathscr{P}_{33}\right) \lambda$ can occur only in the expansions of $\left(y_{3}^{*}\right)^{3} \lambda$ and $\left(y_{3}^{*}\right)^{2} y_{1} Y_{2} \lambda$; this gives $-12\left(y_{3} \mathscr{P}_{33}\right) \lambda$ in all, so that $b=2$. This yields the operator

$$
\begin{aligned}
\varphi_{D^{\prime} A B C} \rightarrow \nabla_{\left(A^{\prime}\right.}^{A} \nabla_{B^{\prime}}^{B} \nabla_{C^{\prime}}^{C} \varphi_{\left.D^{\prime}\right) A B C} & +2\left(\nabla_{\left(A^{\prime}\right.}^{A} \Phi_{B^{\prime} C^{\prime}}^{B C}\right) \lambda_{\left.D^{\prime}\right) A B C} \\
& +4 \Phi_{\left(A^{\prime} B^{\prime}\right.}^{A B} \nabla_{C^{\prime}}^{C} \lambda_{\left.D^{\prime}\right) A B C} .
\end{aligned}
$$

6.6. Powers of the Laplacian. By the general theory, there is a conformally invariant differential operator in $2 n$ dimensions:

$$
\square^{q}: \stackrel{0-2+q 0}{\longrightarrow} \rightarrow \stackrel{0-2-q 0}{\longrightarrow}, \quad 1 \leq q \leq n
$$

whose highest order term is $(\nabla \cdot \nabla)^{q}$. To calculate the full operator for the case $q=2$ observe that the only possibilities from (31) are

$$
\begin{gathered}
\left(\nabla^{a} \nabla_{a} \Lambda\right) f, \quad\left(\nabla^{a} \Lambda\right) \nabla_{a} f, \quad \Lambda \nabla^{a} \nabla_{a} f, \\
\left(\nabla^{a} \nabla^{b} \Phi_{a b}\right) f, \quad\left(\nabla^{a} \Phi_{a b}\right) \nabla^{b} f, \quad \Phi_{a b} \nabla^{a} \nabla^{b} f .
\end{gathered}
$$

The terms in (33) correspond to the inducing expressions

$$
\begin{aligned}
& \left.\theta_{1}=\left\{\left(y_{1}^{\prime} y_{4}^{\prime}+y_{4}^{\prime} y_{1}^{\prime}-y_{2}^{\prime} y_{3}^{\prime}-y_{3}^{\prime} y_{2}^{\prime}\right) \Lambda\right)\right\} \alpha, \\
& \theta_{2}=\left\{\left(y_{1}^{\prime} \Lambda\right) y_{4}^{\prime}+\left(y_{4}^{\prime} \Lambda\right) y_{1}^{\prime}-\left(y_{2}^{\prime} \Lambda\right) y_{3}^{\prime}-\left(y_{3}^{\prime} \Lambda\right) y_{2}^{\prime}\right\} \alpha, \\
& \theta_{3}=\Lambda\left\{\left(y_{1}^{\prime} y_{4}^{\prime}+y_{4}^{\prime} y_{1}^{\prime}-y_{2}^{\prime} y_{3}^{\prime}-y_{3}^{\prime} y_{2}^{\prime}\right) \alpha\right\} .
\end{aligned}
$$

Then $x_{1}\left(a \theta_{1}+b \theta_{2}+c \theta_{3}\right)=0$ if and only if $b=c$ and $a=0$. A similar calculation for (34) reveals that $\square^{2}$ must be of the form

$$
\begin{aligned}
(\nabla \cdot \nabla)^{2} & +d\left\{\left(\nabla^{2} \lambda\right) \nabla_{a} f+\Lambda \nabla^{a} \nabla_{a} f\right\} \\
& +e\left\{\left(\nabla^{a} \Phi_{a b}\right) \nabla^{b} f+\Phi_{a b} \nabla^{a} \nabla^{b} f\right\} .
\end{aligned}
$$

To determine $d$ and $e$, compute how $(\nabla \cdot \nabla)^{2}$ varies under a rescaling of the metric $g_{a b} \rightarrow \tilde{g}_{a b}=\Omega^{2} g_{a b}$. Formulas for this are given in [32]. If $\Upsilon_{a}=\Omega^{-1} \nabla_{a} \Omega$, where $\nabla_{a}$ is the Levi-Civita connection of $g_{a b}$, then the 
Levi-Civita connection $\widetilde{\nabla}_{a}$ of $\widetilde{g}_{a b}$ is given by

$$
\begin{aligned}
\widetilde{\nabla}_{a} \varphi_{\left(A_{1}^{\prime} \cdots A_{j}^{\prime}\right)\left(A_{1} \cdots A_{k}\right)} & =\nabla_{A A^{\prime}} \varphi_{\left(A_{1}^{\prime} \cdots A_{j}^{\prime}\right)\left(A_{1} \cdots A_{k}\right)}-\Upsilon_{A A_{1}^{\prime}} \varphi_{\left(A^{\prime} A_{2}^{\prime} \cdots A_{j}^{\prime}\right)\left(A_{1} \cdots A_{k}\right)} \\
& -\cdots-\Upsilon_{A A_{n}^{\prime}} \varphi_{\left(A_{1}^{\prime} \cdots A_{n-1}^{\prime} A^{\prime} A_{n+1}^{\prime} \cdots A_{j}^{\prime}\right)\left(A_{1} \cdots A_{k}\right)} \\
& \cdots-\Upsilon_{A A_{j}^{\prime}} \varphi_{\left(A_{1}^{\prime} \cdots A_{j-1} A^{\prime}\right)\left(A_{1} \cdots A_{k}\right)} \\
& -\Upsilon_{A_{1} A^{\prime}} \varphi_{\left(A_{1}^{\prime} \cdots A_{j}^{\prime}\right)\left(A A_{2} \cdots A_{k}\right)} \\
& -\cdots-\Upsilon_{A_{n} A^{\prime}} \varphi_{\left(A_{1}^{\prime} \cdots A_{j}^{\prime}\right)\left(A_{1} \cdots A_{n-1} A A_{n+1} \cdots A_{k}\right)} \\
& -\cdots-\Upsilon_{A_{k} A^{\prime}} \varphi_{\left(A_{1}^{\prime} \cdots A_{j}^{\prime}\right)\left(A_{1} \cdots A_{k-1} A\right)},
\end{aligned}
$$

whilst

$$
\begin{gathered}
\widetilde{\Phi}_{A^{\prime} B^{\prime}}^{A B}=\Phi_{A^{\prime} B^{\prime}}^{A B}-\nabla_{\left(A^{\prime}\right.}^{(A} \Upsilon_{\left.B^{\prime}\right)}^{B}+\Upsilon_{\left(A^{\prime}\right.}^{(A} \Upsilon_{\left.B^{\prime}\right)}^{B)}, \\
\widetilde{\Lambda}=\Omega^{-2}\left\{\Lambda+\frac{1}{4} \nabla^{a} \Upsilon_{a}+\frac{1}{4} \Upsilon^{a} \Upsilon_{b}\right\}
\end{gathered}
$$

Employing these in (35), and ignoring all terms but those of the form $\Upsilon \Upsilon \Upsilon \nabla f$, yield $d=e=4$ so that

$$
\square^{2} f=\nabla_{a}\left(\nabla^{a} \nabla^{b}+4 \Phi^{a b}+4 g^{a b} \Lambda\right) \nabla_{b} f .
$$

Remark 6.6.1. This quartic differential operator may be found in [30], [37], [18]; the last reference inspired the work of Eastwood and Rice and this paper.

\section{Conclusions}

To conclude we shall remark briefly on two consequences of the above theory.

7.1. An algebra of invariants. Let $\lambda$ be dominant for $\mathbf{g}$ and $\Upsilon$ let $\mathscr{R}_{\lambda}^{i}=\bigoplus_{w \in W^{\mathbf{p}} ; l(w)=i} \mathscr{O}_{\mathbf{p}}(w \cdot \lambda)$ on $G / P$. There are invariant differential operators between subsheaves of $\mathscr{R}_{\lambda}^{i}$ and $\mathscr{R}_{\lambda}^{i+1}$, and it is always possible to add these with appropriate signs to give differentials $d^{i}: \mathscr{R}_{\lambda}^{i} \rightarrow \mathscr{R}_{\lambda}^{i+1}$ so that $\left(\mathscr{R}^{\bullet}, d^{\bullet}\right)$ is a resolution of $(F(\lambda))^{*}$, i.e.,

$$
0 \rightarrow(F(\lambda))^{*} \rightarrow \mathscr{R}_{\lambda}^{\bullet}
$$

is exact. This resolution (in its algebraic form for Verma modules) is called the Bernstein-Gelfand-Gelfand resolution [5], [28]. Whilst the existence of $\mathscr{R}_{\lambda}^{\bullet}$ and $d^{\bullet}$ depend only on the structure of Verma modules as pmodules; the fact that (36) is a complex depends on the structure of $\mathscr{U}(\mathbf{g})$ 
as an algebra. So sequences such as (36) will exist over a generic curved conformal manifold, but may no longer be a complex. The composition $d^{i+1} \circ d^{i}$ and, indeed, compositions

$$
\mathscr{O}_{\mathbf{p}}\left(w_{1} \cdot \lambda\right) \rightarrow \mathscr{O}_{\mathbf{p}}\left(w_{2} \cdot \lambda\right) \rightarrow \mathscr{O}_{\mathbf{p}}\left(w_{3} \cdot \lambda\right),
$$

with $l\left(w_{3}\right)=l\left(w_{2}\right)+1=l\left(w_{1}\right)+2$, are new invariants of the conformal structure. These depend on the extent to which

$$
\omega^{-1}: \mathbf{g} \rightarrow \Gamma\left(\mathscr{G}, \Theta_{g}\right)
$$

is not a homomorphism of Lie algebras and hence on the conformal curvature.

Example 7.1.1. In four dimensions, let $\lambda=\underset{1}{1} 000, w_{1}=\mathrm{id}, w_{2}=\sigma_{2}$, and $w_{3}=\sigma_{2} \sigma_{3}$, where $\sigma_{i}=\sigma_{\alpha_{i}}$, and simple roots are labelled $\stackrel{\alpha_{1} \alpha_{2} \alpha_{3}}{\longrightarrow}$. Then there are invariant differential operators

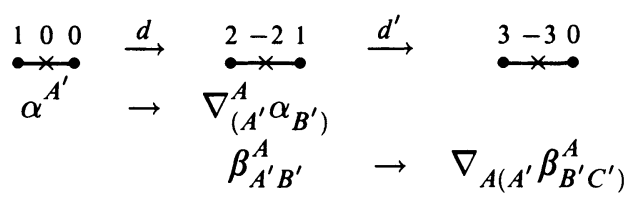

induced by $\beta \rightarrow y_{1} \alpha$ and $\gamma \rightarrow\left(-y_{3}+y_{1} Y_{2}\right) \beta$ for $\alpha, \beta$, and $\gamma$ appropriate highest weight vectors. On $\mathscr{G}$, the composition of these two operators is induced by

$$
\left(-y_{3}^{*}+y_{1}^{*} Y_{2}\right) y_{1}^{*} \alpha=\left[y_{1}^{*}, y_{3}^{*}\right] \alpha .
$$

Letting $\widetilde{\psi}$ induce the self-dual Weyl curvature $\widetilde{\psi}_{A^{\prime} B^{\prime} C^{\prime} D^{\prime}}$ (as $\Phi$ induces tracefree Ricci curvature) it is easy to check that

$$
\left[y_{1}^{*}, y_{3}^{*}\right]=2 \widetilde{\psi} Y_{1}-\frac{1}{2}\left(Y_{1} \widetilde{\psi}\right) h_{1}=\frac{1}{6}\left(Y_{1}^{2} \widetilde{\psi}\right) X_{1}+V,
$$

where $V$ is a function on $\mathscr{G}$ taking values in $\mathbf{u}$. So

$$
\left[y_{1}^{*}, y_{3}^{*}\right] \alpha=2 \widetilde{\psi}\left(Y_{1} \alpha\right)-\frac{1}{2}\left(Y_{1} \widetilde{\psi}\right) \alpha
$$

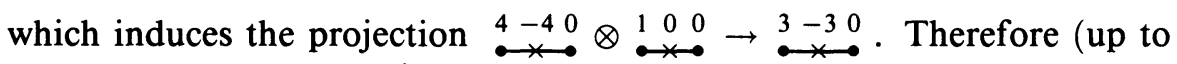
scale) the composition $d^{\prime} \circ d$ consists of tensoring with the self-dual Weyl curvature followed by projection. (This is evident anyway from the formulas for $d$ and $d^{\prime}$.) A similar calculation yields the anti-self-dual Weyl curvature and the Weyl curvature, $W$, in higher dimensions as invariants of the conformal structure.

For further examples of this see [13].

7.2. A Bach tensor in even dimensions. There is a natural analogue of the Bach tensor of four dimensions in even dimension $2 n$. This is obtained by viewing the formal jet of the Weyl tensor as a element of a 
dual Verma module and decomposing under the action of the appropriate parabolic p. To avoid the slight speciality of six dimensions suppose $2 n \geq 8$.

Let $\lambda$ be the highest root of $\mathbf{g}$; so

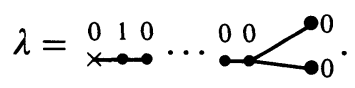

Label the simple roots of $\mathbf{g}$ from left to right as in the appendix and let $w^{0}$ be the longest element of $W^{\mathbf{p}}$. Then the Weyl curvature $W$ is a section of $\mathscr{O}_{\mathbf{p}}\left(\sigma_{1} \sigma_{2} \cdot \lambda\right)$ and there are invariant differential operators

$$
\cdots \rightarrow \mathscr{O}_{\mathbf{p}}\left(\sigma_{1} \cdot \lambda\right) \rightarrow \mathscr{O}_{\mathbf{p}}\left(\sigma_{1} \sigma_{2} \cdot \lambda\right) \stackrel{d}{\longrightarrow} \mathscr{O}_{\mathbf{p}}\left(\sigma_{1} \sigma_{2} \sigma_{3} \cdot \lambda\right) \rightarrow \cdots
$$

corresponding at the level of Verma modules to the p-module extension

$$
0 \rightarrow \operatorname{im} M_{\mathbf{p}}\left(\sigma_{1} \sigma_{2} \sigma_{3} \cdot \lambda\right) \rightarrow M_{\mathbf{p}}\left(\sigma_{1} \sigma_{2} \cdot \lambda\right) \rightarrow N \rightarrow 0
$$

where $N$ is the image of $M_{\mathbf{p}}\left(\sigma_{1} \sigma_{2} \cdot \lambda\right)$ in $M_{\mathbf{p}}\left(\sigma_{1} \cdot \lambda\right)$.

Now $d W$ is the projection of $\nabla_{[a} W_{b c] d e}$ into $\mathscr{O}_{\mathrm{p}}\left(\sigma_{1} \sigma_{2} \cdot \lambda\right)$ and it is easy to check that this coincides with the projection of $\nabla_{[a} R_{b c] d e}$ and is hence zero by the Bianchi identity. Thus if we let $\widehat{I} \subset A(\mathbf{g}) \bigotimes_{\mathscr{Q}^{\prime}(\mathbf{p})} F_{\mathbf{p}}\left(\sigma_{1} \sigma_{2} \cdot \lambda\right)$ cover $I$, then the image of $\widehat{I}$ in the differential operators on $\mathscr{O}\left(\sigma_{1} \sigma_{2} \cdot \lambda\right)$ annihilates $W$.

On the other hand, as a p-module, $N$ is a sum of irreducible quotients of Verma modules [9]

$$
N=L\left(w^{0} \sigma_{1} \cdot \lambda\right) \oplus L\left(\sigma_{1} \sigma_{2} \cdot \lambda\right) .
$$

So there is an element $\beta \in A(\mathbf{g}) \otimes_{\mathscr{U}(\mathbf{p})} F_{\mathbf{p}}\left(\sigma_{1} \sigma_{2} \cdot \lambda\right)$ maximal modulo $\widehat{I}$ which lifts a maximal element of $L\left(w^{0} \sigma_{1}\right)$. By the previous paragraph the differential operator this induces, when applied to the Weyl tensor, yields a new conformally invariant tensor, $B_{a b}$, which is symmetric and tracefree with conformal weight $-2 n+2$, i.e., a section of

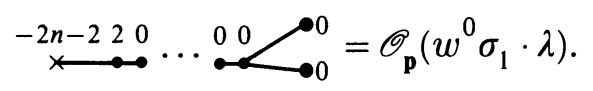

$B_{a b}$ depends on the derivatives of a metric in the conformal class up to order $2 n$. A similar argument works in six dimensions, and in four dimensions produces the ordinary Bach tensor.

Remark 7.2.1. A similar tensor occurs in the work of Fefferman and Graham [20]; it is almost certainly the same tensor. On the other hand, 
$B_{a b}$ is evidently not the fourth order differential operator proposed as a generalization of the Bach tensor in [10], [37].

\section{Appendix}

This appendix summarizes the basic structure and representation theory of semisimple Lie algebras which is required in this paper.

A.1. Structure theory of semisimple Lie algebras. Let $\mathrm{g}$ be a semisimple complex Lie algebra with a distinguished Cartan subalgebra $\mathbf{h}$ and set of roots $\Delta(\mathbf{g}, \mathbf{h})$. The root space decomposition of $\mathbf{g}$ is

$$
\mathbf{g}=\mathbf{h} \oplus\left(\underset{\alpha \in \Delta(\mathbf{g}, \mathbf{h})}{\oplus} \mathbf{g}_{\alpha}\right) .
$$

Fix a set $\mathscr{S}$ of simple roots in $\Delta(\mathbf{g}, \mathbf{h})$ and let $\Delta^{+}(\mathbf{g})$ be the corresponding set of positive roots. Then any maximal solvable subalgebra of $\mathbf{g}$ is conjugate to the standard Borel algebra

$$
\mathbf{b}=\mathbf{h} \oplus\left(\underset{\alpha \in \Delta^{+}(\mathbf{g})}{\oplus} \mathbf{g}_{\alpha}\right) .
$$

Let $\mathscr{S}_{\mathbf{p}} \subset \mathscr{S}$ and let $\Delta(\mathbf{l})$ be the span of $\mathscr{S}_{\mathbf{p}}$ in $\Delta(\mathbf{g}, \mathbf{h})$ and $\Delta(\mathbf{u})=$ $\Delta^{+}(\mathbf{g}) \backslash \Delta(\mathbf{l})$. So define subalgebras

$$
\mathbf{l}=\mathbf{h} \oplus\left(\underset{\alpha \in \Delta(\mathbf{l})}{\oplus} \mathbf{g}_{\alpha}\right), \quad \mathbf{u}=\underset{\alpha \in \Delta(\mathbf{u})}{\oplus} \mathbf{g}_{\alpha},
$$

and

$$
\mathbf{p}=\mathbf{l} \oplus \mathbf{u} .
$$

Any parabolic subalgebra of $\mathbf{g}$ is conjugate to such a $\mathbf{p}$ for an appropriate choice of $\mathscr{S}_{\mathbf{p}}$.

The Cartan-Killing form on $\mathbf{g}$ induces a bilinear form $\langle$,$\rangle on \mathbf{h}^{*}$. Restricted to the real span $\mathbf{h}_{\mathbf{R}}^{*}$ of the simple roots this form is positive definite. For any root $\alpha$ define its coroot by

$$
\alpha^{\vee}=\frac{2 \alpha}{\langle\alpha, \alpha\rangle}
$$

Then for $\alpha_{i} \in \mathscr{S}$ the Cartan matrix of integers $\left\langle\alpha_{i}, \alpha_{j}^{\vee}\right\rangle$ determine and are determined by $\mathbf{g}$. They are recorded by means of a Dynkin diagram for $\mathbf{g}$. Recall that this is a partially directed graph with a node for each simple root and edges determined by the Cartan integers according to the following:

1. $\left\langle\alpha_{i}, \alpha_{j}^{\vee}\right\rangle=2$. 
2. $\alpha_{i} \neq \alpha_{j}$ are connected iff $\left\langle\alpha_{i}, \alpha_{j}^{\vee}\right\rangle \neq 0$.

3. $\stackrel{\alpha \beta}{\leftrightarrow} \Leftrightarrow\left\langle\alpha, \beta^{\vee}\right\rangle=-1$,

$$
\begin{aligned}
& \stackrel{\alpha}{\Rightarrow} \Leftrightarrow\left\langle\alpha, \beta^{\vee}\right\rangle=-2,\left\langle\beta, \alpha^{\vee}\right\rangle=-1, \\
& \stackrel{\alpha}{\Leftrightarrow} \Leftrightarrow\left\langle\alpha, \beta^{\vee}\right\rangle=-3,\left\langle\beta, \alpha^{\vee}\right\rangle=-1 \text {. }
\end{aligned}
$$

To specify a standard parabolic subalgebra of $\mathbf{g}$ by giving $\mathscr{S}_{\mathbf{p}}$ it will be convenient to give the Dynkin diagram for $\mathbf{g}$ with the nodes corresponding to simple roots of $\mathbf{g}$ not in $\mathscr{S}_{\mathbf{p}}$ crossed through.

Example A.1.1. The conformal motions of the complex sphere of dimension $m \geq 3$ are given by $\mathrm{SO}(m+2, \mathbf{C})$. The stabilizer of a point is a subgroup isomorphic to the conformal Poincaré group $P=(\mathrm{SO}(m, \mathbf{C}) \times$ $\left.\mathbf{C}^{\times}\right) \ltimes \mathbf{C}^{m}$. Then, for $m \geq 5$,

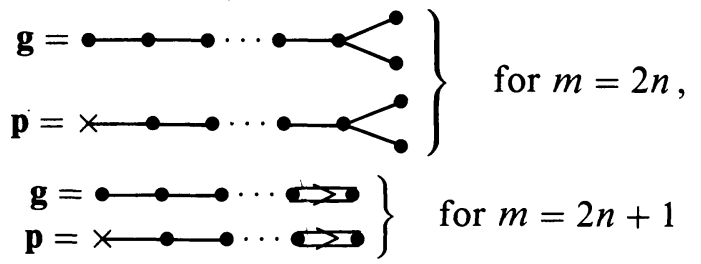

(both diagrams have $n+1$ nodes). The case $m=4$ is special, for so $(6, \mathbf{C}) \cong \operatorname{sl}(4, m \mathbf{C})$. So

$$
\mathbf{g}=\bullet \text { and } \mathbf{p}=\bullet \longleftrightarrow \text {. }
$$

A.2. Representation theory. Let $F$ be a finite-dimensional irreducible representation of $\mathbf{g}$. Under the action of $\mathbf{h}, F$ decomposes as a direct sum of simultaneous eigenspaces or weight spaces:

$$
F=\bigoplus_{\lambda \in \Delta(F)} F_{\lambda}, \quad \text { where } F_{\lambda}=\{f \in F \mid h \cdot f=\lambda(h) f \forall h \in \mathbf{h}\} .
$$

Those $\lambda \in \mathbf{h}^{*}$ such that $F_{\lambda} \neq\{0\}$ are called the weights of $F$. Amongst these there is an unique one, $\lambda$, such that $\left\langle\lambda, \alpha^{\vee}\right\rangle$ is a nonnegative integer for all $\alpha \in \mathscr{S}$, and this correspondence between irreducible representations and such dominant integral weights classifies all finite-dimensional irreducible representations. Denote by $F(\lambda)$ the irreducible g-modules with highest weight $\lambda$.

Now let $F$ be a finite-dimensional irreducible representation of $\mathbf{p}$. Let $\mathbf{l}$ and $\mathbf{u}$ be as in the Levi decomposition (37) of $\mathbf{p}$ so that $\mathbf{l}$ is reductive and $\mathbf{u}$ is nilpotent. By Engel's theorem [23] $\mathbf{u}$ acts on $F$ by nilpotent endomorphisms and hence, by irreducibility, by zero. $F$ is therefore a trivial extension of a finite-dimensional irreducible representations of $\mathbf{l}$. Now

$$
\mathbf{l}=\mathbf{l}^{s} \oplus \mathbf{l}_{z}
$$


where $\mathbf{l}^{s}=[\mathbf{l}, \mathbf{l}]$ is semisimple and $\mathbf{l}_{z}$ is the center of $\mathbf{l}$. So an irreducible representation of $\mathbf{l}$ is specified by a dominant integral weight for $\mathbf{l}^{S}$ and an element of $\mathbf{l}_{z}^{*}$. Since it is always possible to arrange that $\mathbf{h}=\mathbf{h} \cap \mathbf{l}^{s} \oplus \mathbf{l}_{z}$, such a representation is specified by a weight $\lambda$ such that $\left\langle\lambda, \alpha^{\vee}\right\rangle$ is a nonnegative integer for all $\alpha \in \mathscr{S}_{\mathrm{p}}$. A weight dominant for $\mathbf{l}^{s}$ is called dominant for $\mathbf{p}$. Denote by $F_{\mathbf{p}}(\lambda)$ the irreducible p-module with highest weight $\lambda$.

In both preceding paragraphs, $F_{\lambda}$ is one-dimensional and maximal, i.e., annihilated by all positive weight spaces of $\mathbf{g}$ (which all occur in $\mathbf{p}$ ). $\lambda$ is highest amongst all weights in $F$ with respect to the usual partial ordering of weights (given $\mathscr{S}) . F_{\lambda}$ generates $F$ under the action of $\mathscr{U}(\mathbf{g})$ or $\mathscr{U}(\mathbf{p})$, respectively.

A weight $\mu \in \mathbf{h}^{*}$ for $\mathbf{g}$ may be indicated by inscribing over a node of the Dynkin diagram of $\mathbf{g}$ corresponding to a simple root $\alpha$ the coefficient $\left\langle\mu, \alpha^{\vee}\right\rangle$. Generally it will be necessary only to consider integral weights, i.e., weights for which these coefficients are integers. If an irreducible representation of $\mathbf{p}$ exponentiates to a representation of the corresponding parabolic subgroup $P$ of $G$, then necessarily its weights are integral. This condition is sufficient if $G$ and hence $P$ are simply connected.

A.3. The Weyl group and parabolic Hasse subgraphs. A hyperplane $W_{\alpha}$ in $\mathbf{h}_{\mathbf{R}}^{*}$ perpendicular to a root $\alpha \in \Delta(\mathbf{g}, \mathbf{h})$ is called a wall; the Weyl group $W_{\mathbf{g}}$ of $\mathbf{g}$ is the group of reflections $\sigma_{\alpha}$ in these walls. It is generated by simple reflections, i.e., reflections $\sigma_{\alpha_{i}}$ in walls $W_{\alpha_{i}}$ for $\alpha_{i} \in \mathscr{S}$. Any element $w$ of the Weyl group has a minimal length expression in terms of simple reflections; the length $l(w)$ of $w$ is the length of such an expression.

It is useful to have a means of realizing the action of a simple reflection on a weight, expressed in the above notation:

Recipe for the action of a simple reflection.

To compute $\sigma_{\alpha}(\lambda)$, let $c$ be the coefficient of the node associated to $\alpha$. Add $c$ to the adjacent coefficients, with multiplicity if there is a multiple edge directed towards the adjacent node, and, finally, replace $c$ by $-c$.

Example A.3.1. Apply the reflection $\sigma_{\alpha}$ in the simple root $\alpha$ indicated by the $\uparrow$ :

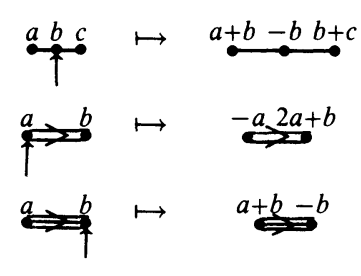


The Weyl group $W_{\mathbf{g}}$ admits the structure of a directed graph as follows: let $w, w^{\prime} \in W_{\mathbf{g}}$ and set $w \rightarrow w^{\prime}$ if $l\left(w^{\prime}\right)=l(q)+1$ and $w^{\prime}=\sigma_{\alpha} w$ for some $\alpha \in \Delta(\mathbf{g})$. Set no other edges in $W_{\mathbf{g}}$. This directed graph structure gives a partial ordering [8]: we write $w \preceq w^{\prime}$ if either $w=w^{\prime}$ or there exists a directed path from $w$ to $w^{\prime}$ in $W(\mathbf{g})$.

Remark A.3.2. For a discussion and examples of the directed graph structure of Weyl groups, see [22], [5].

For the purposes of this paper, a subgraph of the Weyl group associated to a parabolic subalgebra is more important than the Weyl group itself. This subgraph, $W^{\mathbf{p}}$, is the subset of $W_{\mathbf{g}}$ whose reflection action sends a weight $\lambda$, dominant for $\mathbf{g}$, to a weight dominant for $\mathbf{p}$. Define

$$
\Delta(w)=\left\{\alpha \in \Delta^{+}(\mathbf{g}) ; w^{-1} \alpha \in-\Delta^{+}(\mathbf{g})\right\} .
$$

Then it can be shown that

$$
W^{\mathbf{p}}=\left\{w \in W_{\mathbf{g}} ; \Delta(w) \subset \Delta(\mathbf{u})\right\} .
$$

$|\Delta(w)|=l(w)$ and the subgraph structure is induced on $W^{\mathbf{p}}$. Detailed calculations of $W^{\mathbf{p}}$ may be found in [22], [5]; the following examples suffice for the purposes of this paper.

Example A.3.3. If $\mathbf{p}=\bullet \longleftrightarrow$ then $W^{\mathbf{p}}$ is

$$
\mathrm{id} \rightarrow(2) \nearrow_{(23)}^{(21)} \succ^{\succ^{(213)} \rightarrow(2132)}
$$

Here, $(i j \cdots k)=\sigma_{\alpha_{i}} \sigma_{\alpha_{j}} \cdots \sigma_{\alpha_{k}}$ and the simple roots of $\mathbf{g}$ are $\alpha_{1}^{\alpha_{2} \alpha_{3}}$.

Example A.3.4. If

$$
\mathbf{P}=\stackrel{\alpha_{1} \alpha_{2} \alpha_{3}}{\longleftrightarrow} \ldots \stackrel{\alpha_{n-2} \alpha_{n-1}}{\longleftrightarrow} \overbrace{}^{\alpha_{n}}
$$

then, in the notation of the previous example, $W^{\mathbf{p}}$ is:

$$
\begin{aligned}
\text { id } \rightarrow(1) & \rightarrow(12) \rightarrow \cdots \\
\rightarrow(12 \cdots(n-1)) & \searrow_{(12 \cdots(n-1)(n+1))}(12 \cdots(n-1)(n)) \searrow_{(12 \cdots(n-1)(n)(n+1))} \\
& \rightarrow(12 \cdots(n-1)(n)(n+1)(n-1)) \rightarrow \cdots \rightarrow(12 \cdots 21)
\end{aligned}
$$

Example A.3.5. If

then $W^{\mathbf{p}}$ is

$$
\mathbf{p}=\stackrel{\alpha_{1} \alpha_{2} \alpha_{3}}{\longleftrightarrow} \cdots \stackrel{\alpha_{n} \alpha_{n+1}}{\rightleftharpoons}
$$

$$
\begin{aligned}
\text { id } \rightarrow(1) \rightarrow(12) \rightarrow \cdots(12 \cdots(n+1)) & \rightarrow(12 \cdots(n+1)(n)) \\
& \rightarrow \cdots \rightarrow(12 \cdots 21) .
\end{aligned}
$$


A.4. Homogeneous bundles. The homogeneous vector bundle over $G / P$ induced by the representation $F^{*}$, where $F=F_{\mathrm{p}}(\lambda)$, has highest weight $\lambda$, or its sheaf of holomorphic sections will be denoted by either $\mathscr{O}_{\mathbf{p}}(\lambda)$ or the Dynkin diagram for $\mathbf{p}$ with the coefficients $\left\langle\lambda, \alpha_{j}\right\rangle$ inscribed over the nodes. Thus, for example, the reduced spinor bundles on the complex spheres of even dimension are

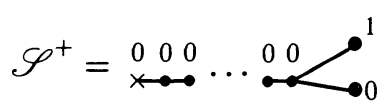

and

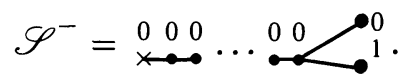

The tangent bundle on this manifold is

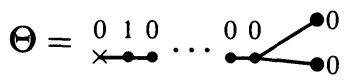

whilst the cotangent bundle is

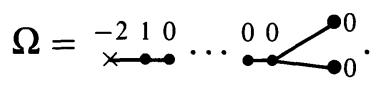

The odd dimensional spheres have a single spinor bundle

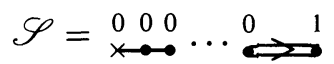

with tangent bundle

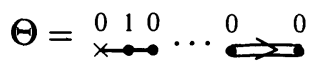

and cotangent bundle

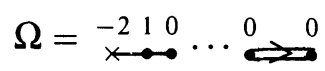

The conformal line bundles are, in even dimensions,

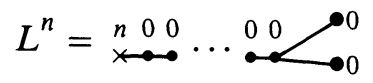

and, in odd dimensions

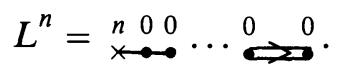

Recall that sections of these bundles may locally be represented by functions $f$ on $X$ which rescale as $f \rightarrow \Omega^{n} f$, when a local choice of metric within the conformal class is rescaled by $g \rightarrow \Omega^{2} g$.

This notation will also denote vector bundles and sheaves similarly induced using the $P$-principal bundle $\mathscr{G} \rightarrow \mathscr{G} / P=X$. 


\section{References}

[1] M. F. Atiyah, R. Bott, \& V. K. Patodi, On the heat equation and the index theorem, Invent. Math. 19 (1973) 279-330.

[2] R. Bach, Zur Weylschen Relativitätstheorie und der Weylschen Erweiterung des Krümmungstensorbegriffs, Math. Z. 9 (1921) 110-135.

[3] R. J. Baston, The algebraic construction of invariant differential operators, thesis, University of Oxford, 1985.

[4] __ Almost Hermitian symmetric spaces I: Local twistor theory (to appear).

[5] R. J. Baston \& M. G. Eastwood, The Penrose transform: Its Interaction with representation theory, Oxford University Press, 1989.

[6] A. Beilinson \& J. Bernstein, Localisation de g-modules, C. R. Acad. Sci. Paris 292 (1981) $15-18$.

[7] I. N. Bernstein, I. M. Gelfand \& S. I. Gelfand, Structure of representations generated by vectors of highest weight, Funct. Anal. Appl. 5 (1971) 1-8.

[8] _ Schubert cells and the cohomology of the spaces $G / P$, in Representation Theory, London Math. Soc. Lecture Note Series, No. 69, Cambridge University Press, 1982.

[9] B. D. Boe \& D. H. Collingwood, A comparison theory for the structure of induced representations. I, J. Algebra 94 (1985) 511-545.

[10] T. Branson, Differential operators canonically associated to a conformal structure, Math. Scand. 57 (1985) 293-345.

[11] J. L. Brylinski \& M. Kashiwara, Kazhdan-Lusztig conjecture and holonomic systems, Invent. Math. 64 (1981) 387-410.

[12] K. Dighton, An introduction to the theory of local twistors, Internat. J. Theoret. Phys. 11 (1974) 31-43.

[13] M. G. Eastwood, The Fefferman Graham conformal invariant, Twistor Newsletter 20 (1985) 46.

[14] _ , The generalized Penrose-Ward transform, Math. Proc. Cambridge Philos. Soc. 97 (1985) 165-187.

[15] __ Real conformal invariants, Twistor Newsletter 24 (1987) 20.

[16] M. G. Eastwood, R. Penrose \& R. O. Wells, Cohomology and massless fields, Comm. Math. Phys. 78 (1981) 305-351.

[17] M. G. Eastwood \& J. W. Rice, Conformally invariant differential operators on Minkowski space and their curved analogues, Comm. Math. Phys. 109 (1987) 207-228.

[18] M. G. Eastwood \& M. A. Singer, Conformally invariant Maxwell gauge, Phys. Lett. A 107 (1985) 73-74.

[19] M. G. Eastwood \& K. P. Tod, Edth-A differential operator on the sphere, Math. Proc. Cambridge Philos. Soc. 92 (1982) 317-330.

[20] C. Fefferman \& C. R. Graham, Conformal invariants, Preprint, Princeton University, Princeton, NJ, 1984.

[21] H. D. Fegan, Conformally invariant first order differential operators, Quart. J. Math. 27 (1976) 371-378.

[22] H. Hiller, Geometry of Coxeter groups, Research Notes in Math., No. 54, Pitman, Boston, 1982.

[23] J. Humphreys, Introduction to Lie algebras and representation theory, Graduate Texts in Math., No. 9, Springer, New York, 1972.

[24] S. Kobayashi, Transformation groups in differential geomeltry, Springer, Berlin, 1972.

[25] B. Kostant, Lie algebra cohomology and the generalized Borel-Weil theorem, Ann. of Math. (2) 74 (1961) 329-387.

[26] _ _ Verma modules and the existence of quasi invariant differential operators, NonCommutative Harmonic Analysis, Lecture Notes in Math., Vol. 466, Springer, Berlin, 1975. 
[27] C. Kozameh, E. T. Newman \& K. P. Tod, Conformal Einstein spaces, General Relativity and Gravitation 17 (1985), 343-352.

[28] J. Lepowsky, A generalization of the Bernstein-Gelfand-Gelfand resolution, J. Algebra 49 (1977) 496-511.

[29] T. Ochiai, Geometry associated with semisimple flat homogeneous spaces, Trans. Amer. Math. Soc. 152 (1970) 159-193.

[30] S. Paneitz, A quartic conformally invariant differential operator for arbitrary pseudoRiemannian manifolds, Preprint, M.I.T., 1983.

[31] R. Penrose \& W. Rindler, Spinors and space time. I, Cambridge University Press, Cambridge, 1984.

[32] __ Spinors and space time. II, Cambridge University Press, Cambridge, 1986.

[33] D. C. Spencer, Overdetermined systems of linear partial differential equations, Bull. Amer. Math. Soc. 75 (1969) 179-139.

[34] N. Tanaka, On the equivalence problems associated with a certain class of homogeneous spaces, J. Math. Soc. Japan 17 (1965) 103-139.

[35] D.-N. Verma, Structure of certain induced representations of complex semisimple Lie algebras, Bull. Amer. Math. Soc. 74 (1968) 160-166.

[36] D. Vogan, Representations of real reductive Lie groups, Progress in Math., No. 15, Birkhäuser, Boston, 1981.

[37] V. Wunsch, On conformally invariant differential operators, Math. Nachr. 129 (1986) 269-281.

UNIVERSITY OF OXFORD 